\title{
Evolution of metastable structures in bimetallic surfaces from microscopy and machine-learning molecular dynamics
}

\author{
$\underline{\text { Jin Soo Lim }}{ }^{1}$, Jonathan Vandermause ${ }^{1}$, Matthijs A. van Spronsen ${ }^{1,3}$, Albert Musaelian ${ }^{1}$, Yu Xie ${ }^{1}$, Lixin Sun ${ }^{1}$, \\ Christopher R. O'Connor ${ }^{1}$, Tobias Egle ${ }^{1}$, Nicola Molinari ${ }^{1}$, Jacob Florian ${ }^{1}$, Kaining Duanmu², \\ Cynthia M. Friend ${ }^{1}$, Robert J. Madix ${ }^{1}$, Philippe Sautet ${ }^{2}$, and Boris Kozinsky ${ }^{1}$
}

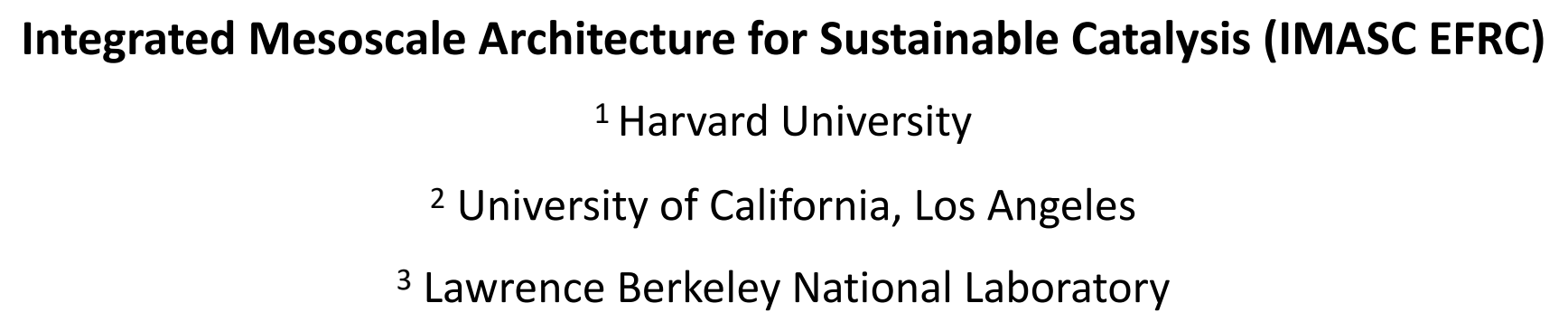

${ }^{3}$ Lawrence Berkeley National Laboratory 


\section{Rational catalyst design}

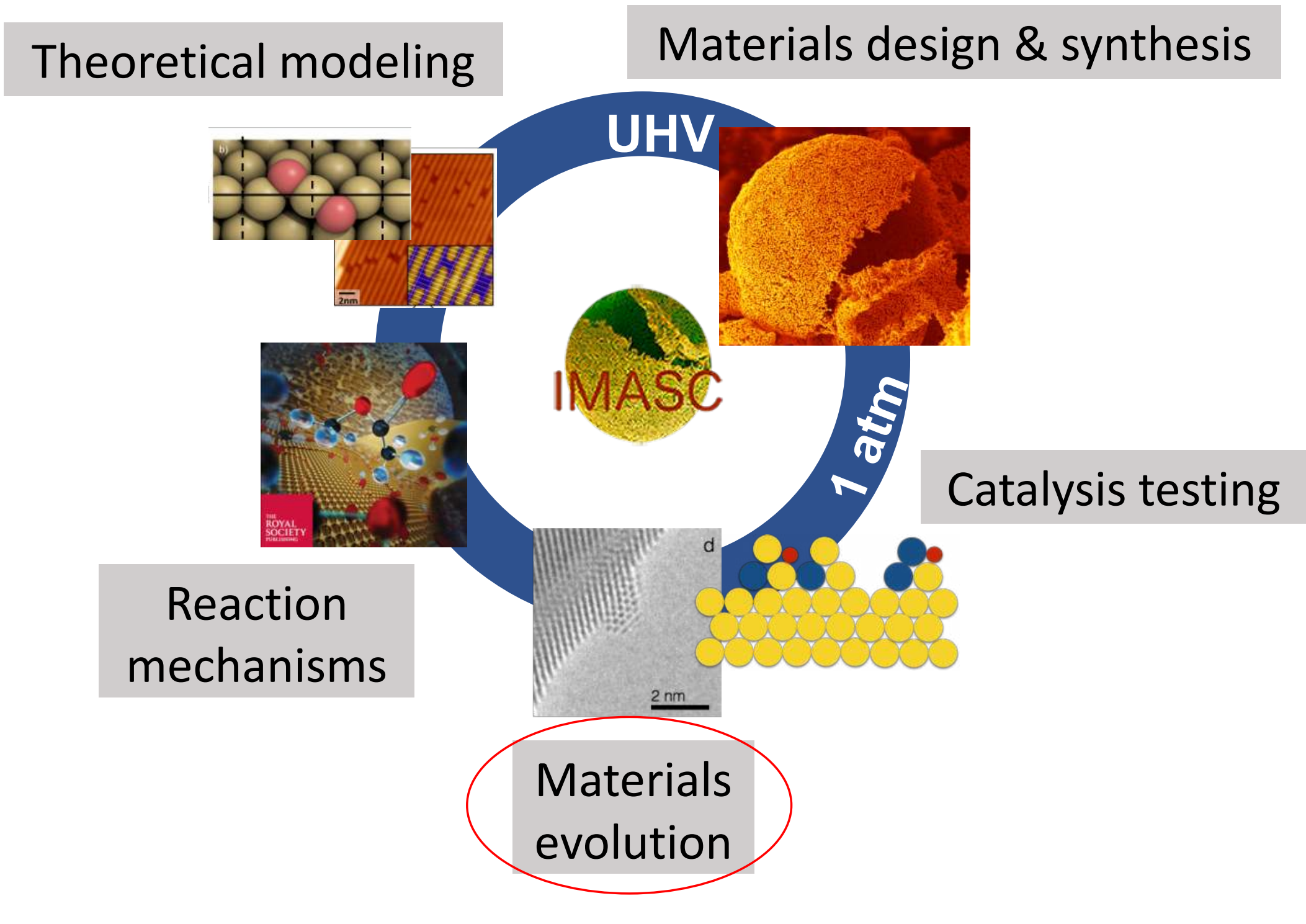




\section{Dilute bimetallic catalysts}

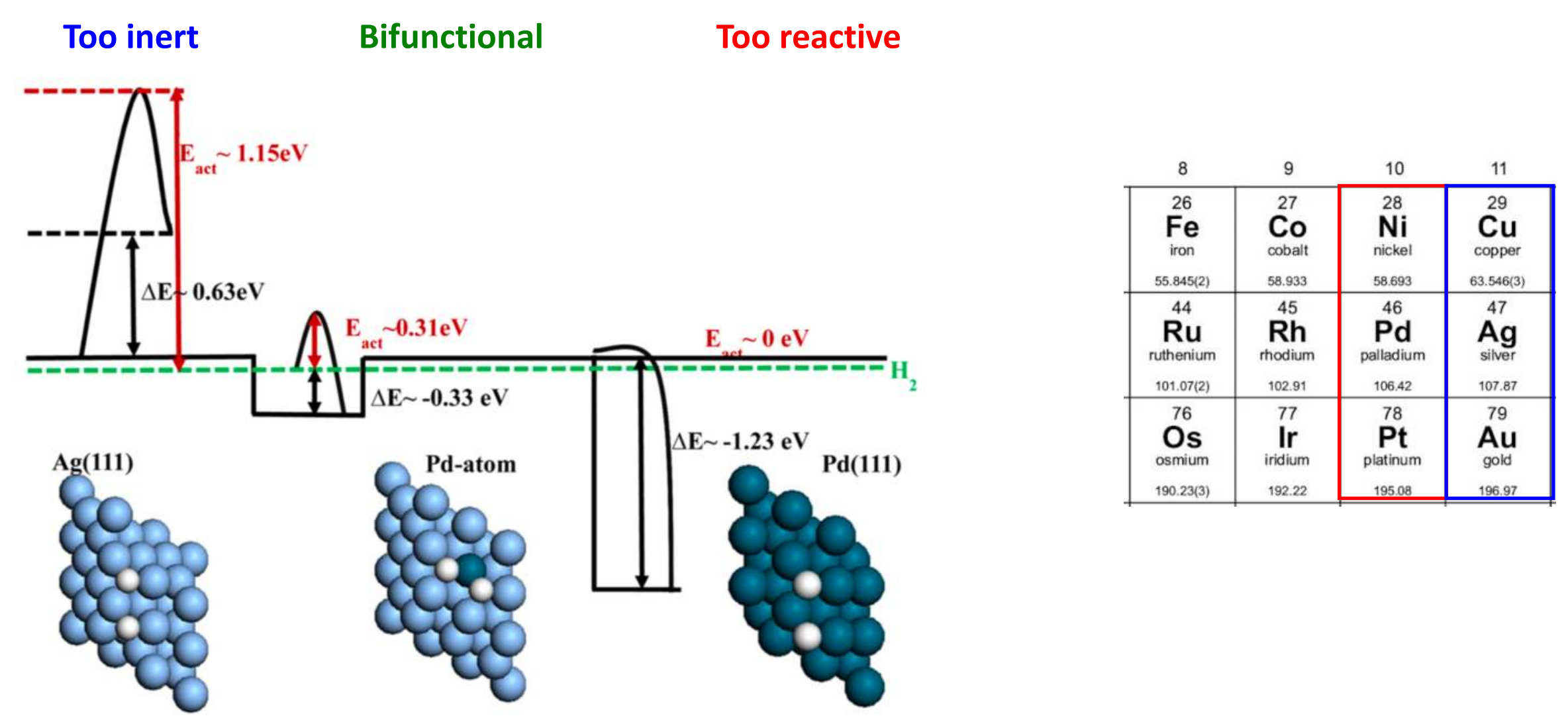

J. Phys. Chem. C 119, (2015) 18140-18148. 


\section{Surface segregation}

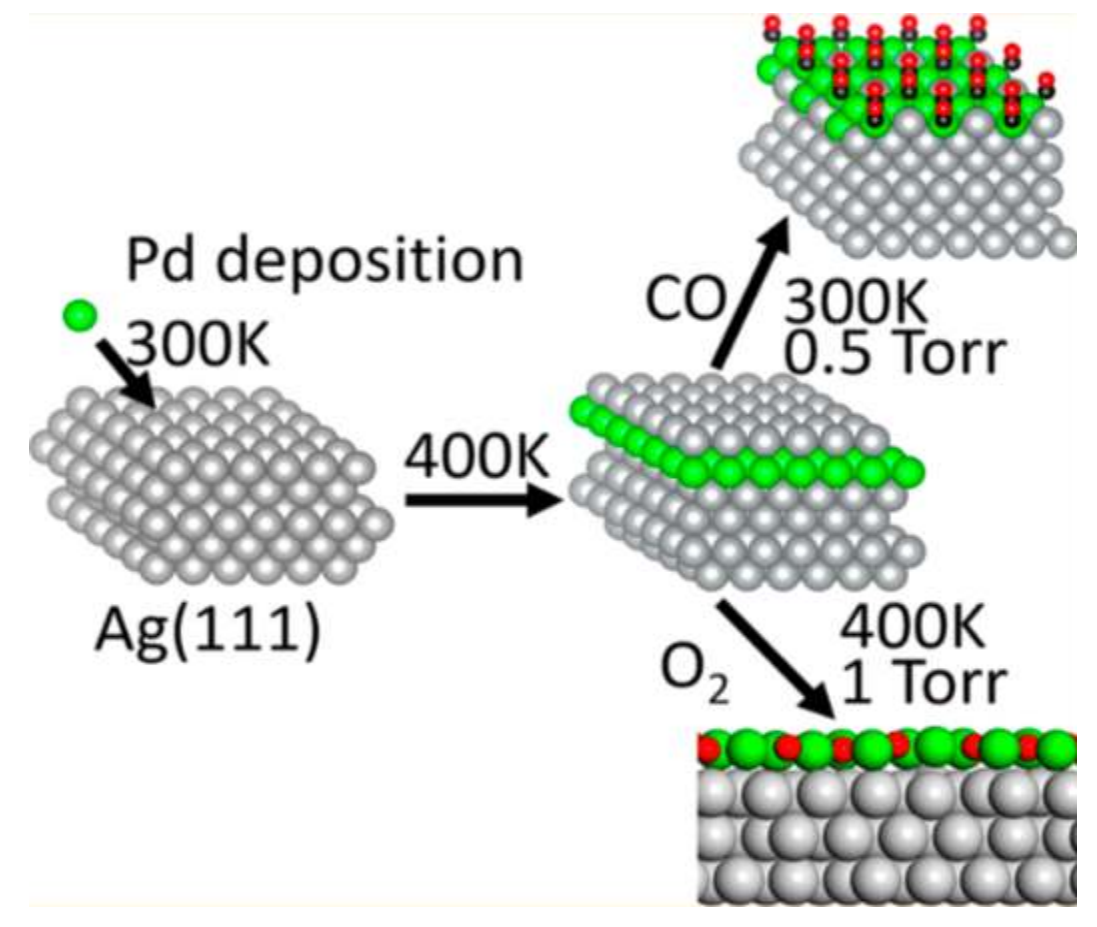

J. Phys. Chem. C 123, (2019) 8312-8323.

\section{Pd dissolution}

Ag surface segregation in vacuum

$\downarrow$

Adsorbate-induced

$\mathrm{Pd}$ reverse segregation 


\section{$\mathrm{Pd} / \mathrm{Ag}$ heteroepitaxy}

\section{"Surfactant" effect Layer-by-layer dissolution}

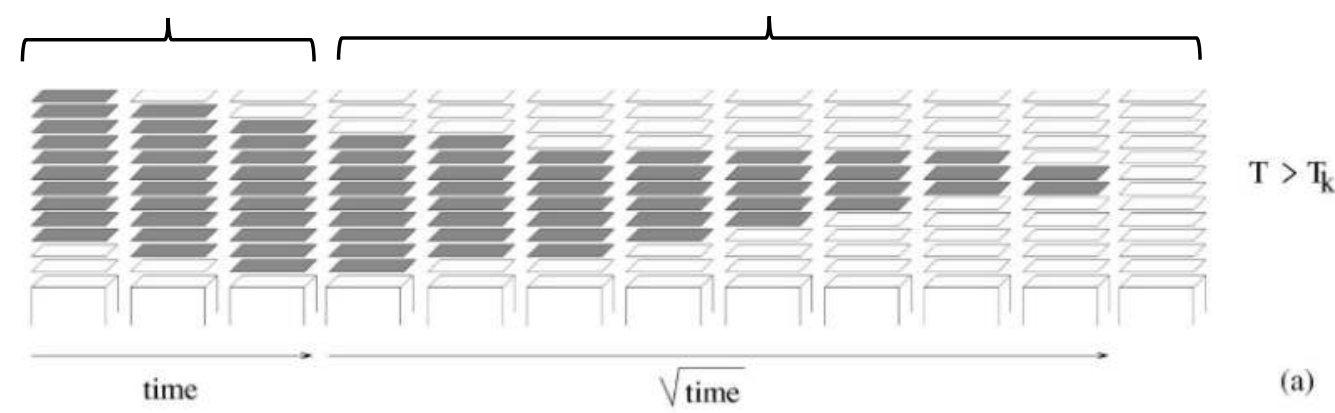

Phys. Rev. B 60, (1999) 13890-13801.

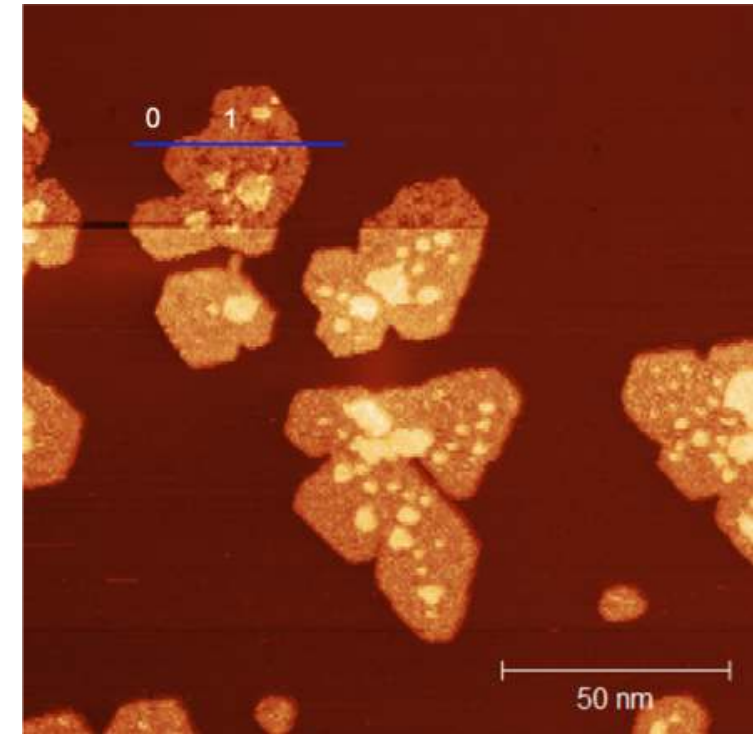

$0.2 \mathrm{ML} \mathrm{Pd} / \mathrm{Ag}(111)$

$300 \mathrm{~K}$

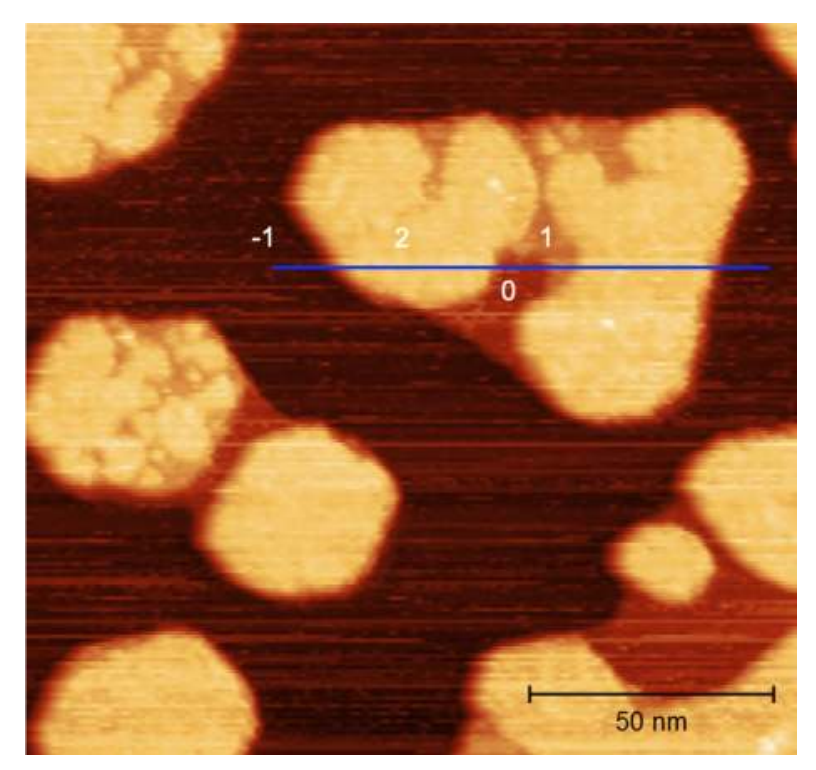

Ag encapsulation 


\section{Automated modeling strategy}
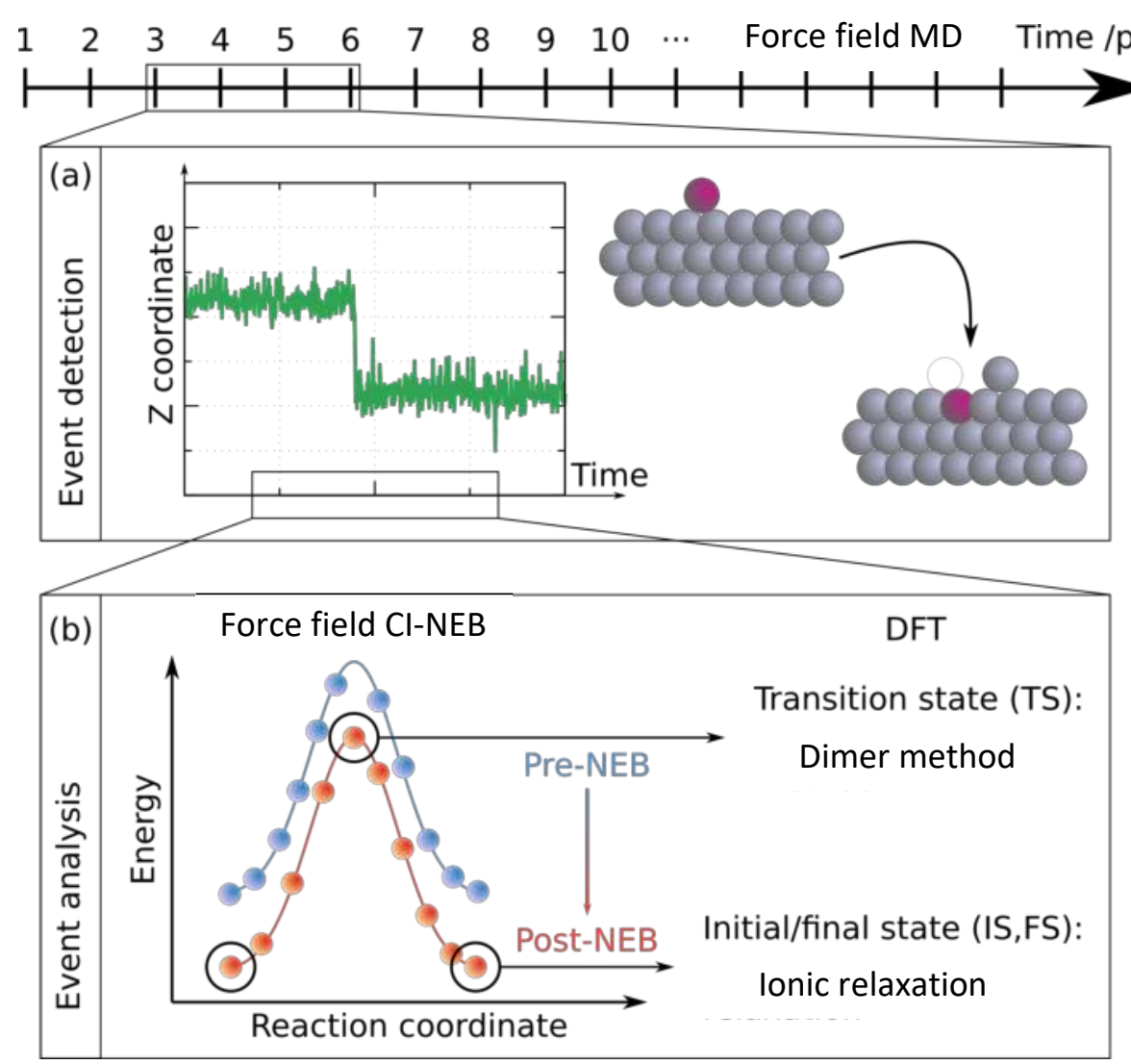

1) High-temperature force field MD

2) Automated detection of restructuring events

3) DFT optimization

J. Phys. Chem. C 123, (2019) 16332-16344. 


\section{Restructuring events: $\mathrm{Pd} / \mathrm{Ag}$ single-atom alloy}

(a)

IS

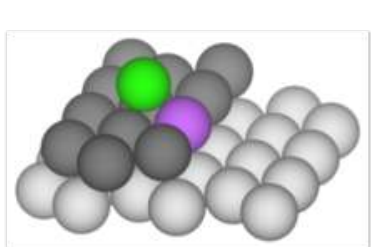

(b)

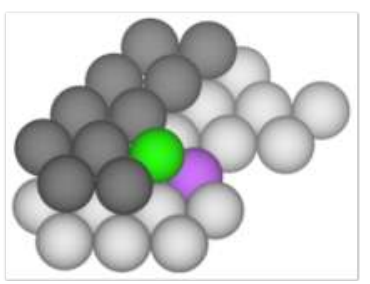

(c)

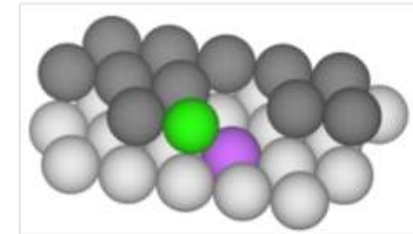

TS
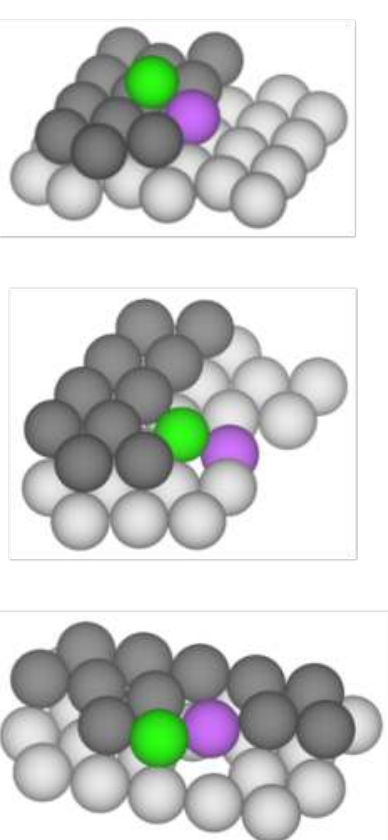

MS

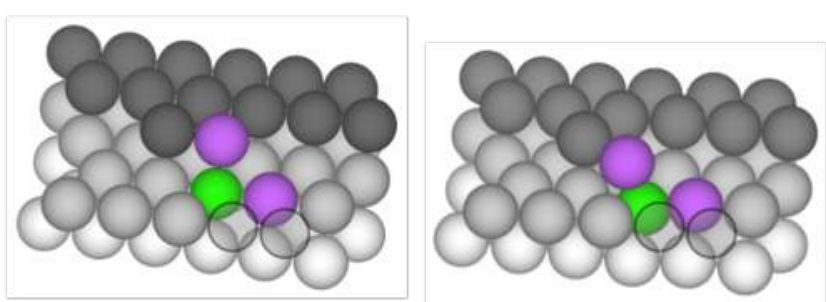

(a) Exchange descent

(b) Vacancy insertion

(c) Direct exchange

(d) Interlayer exchange (d)

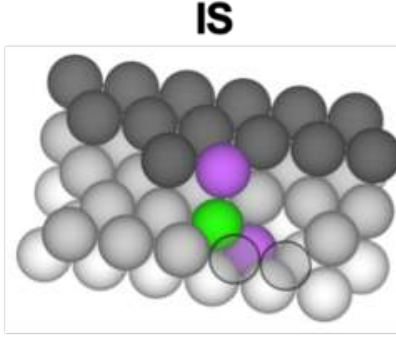

TS1

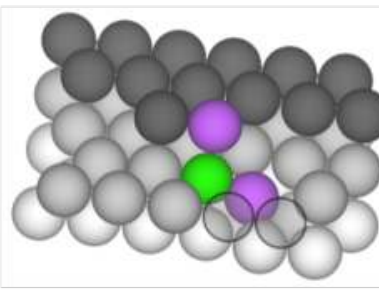

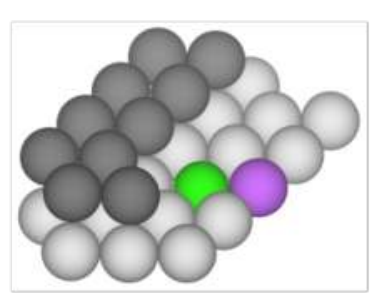

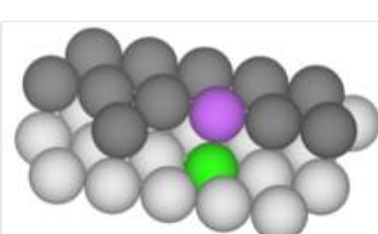

TS2

FS

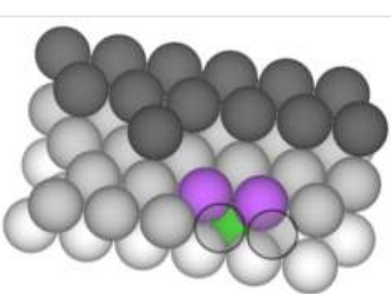

- $\mathrm{Ag}$

Pd in motion

Auxiliary Ag 


\section{Gaussian process machine learning force field}

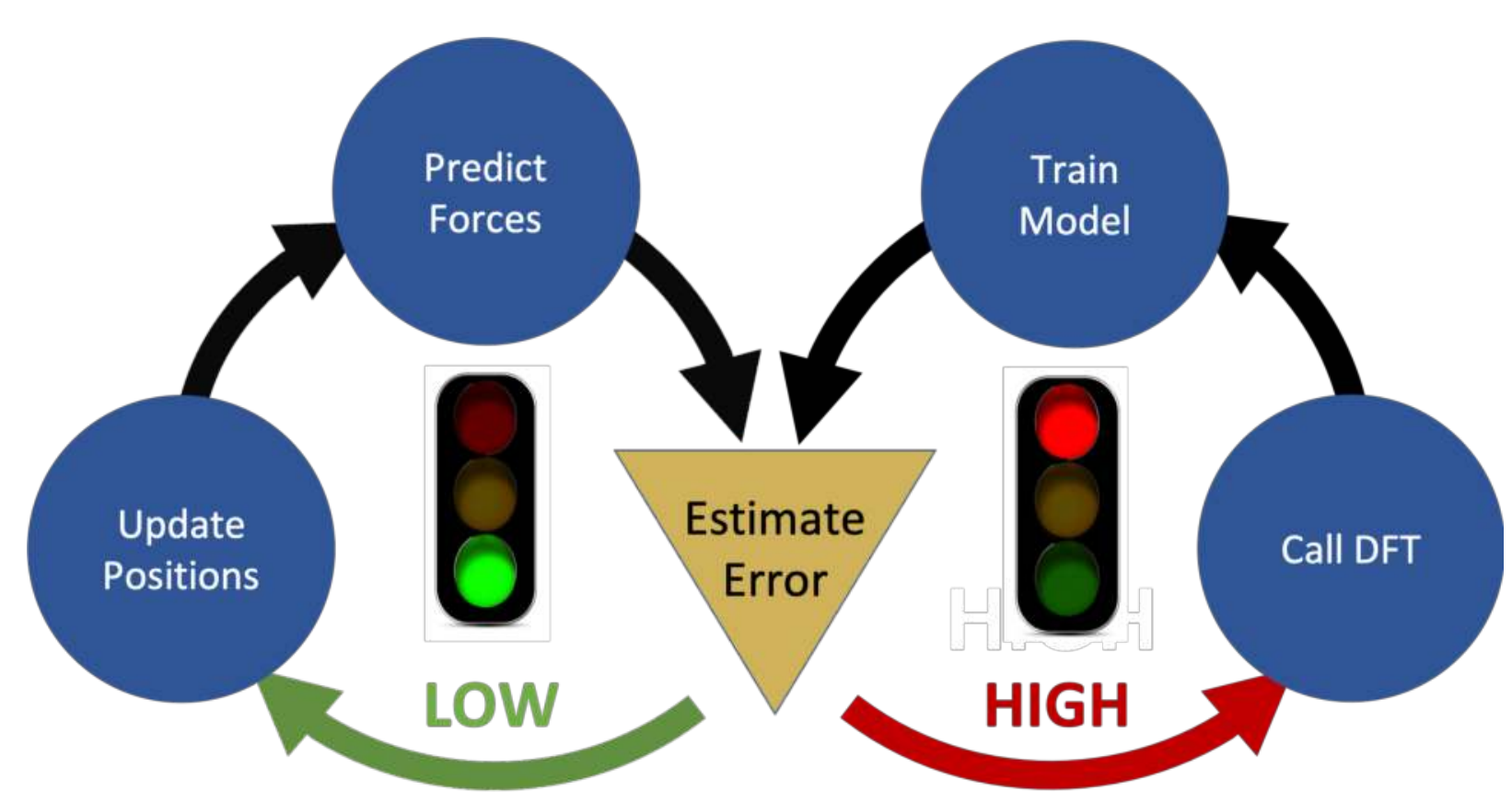

Fast Learning of Atomistic Rare Events (FLARE)

arXiv:1904.02042 (2019)

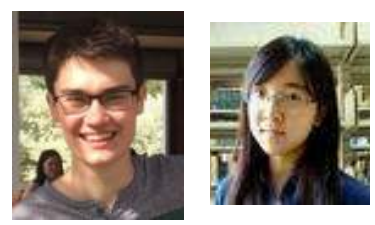

Gaussian process Bayesian active learning

- Automated \& minimalistic training

- Request DFT data based on error estimation

- Mapped to cubic spline models 


\section{Fully automated workflow}

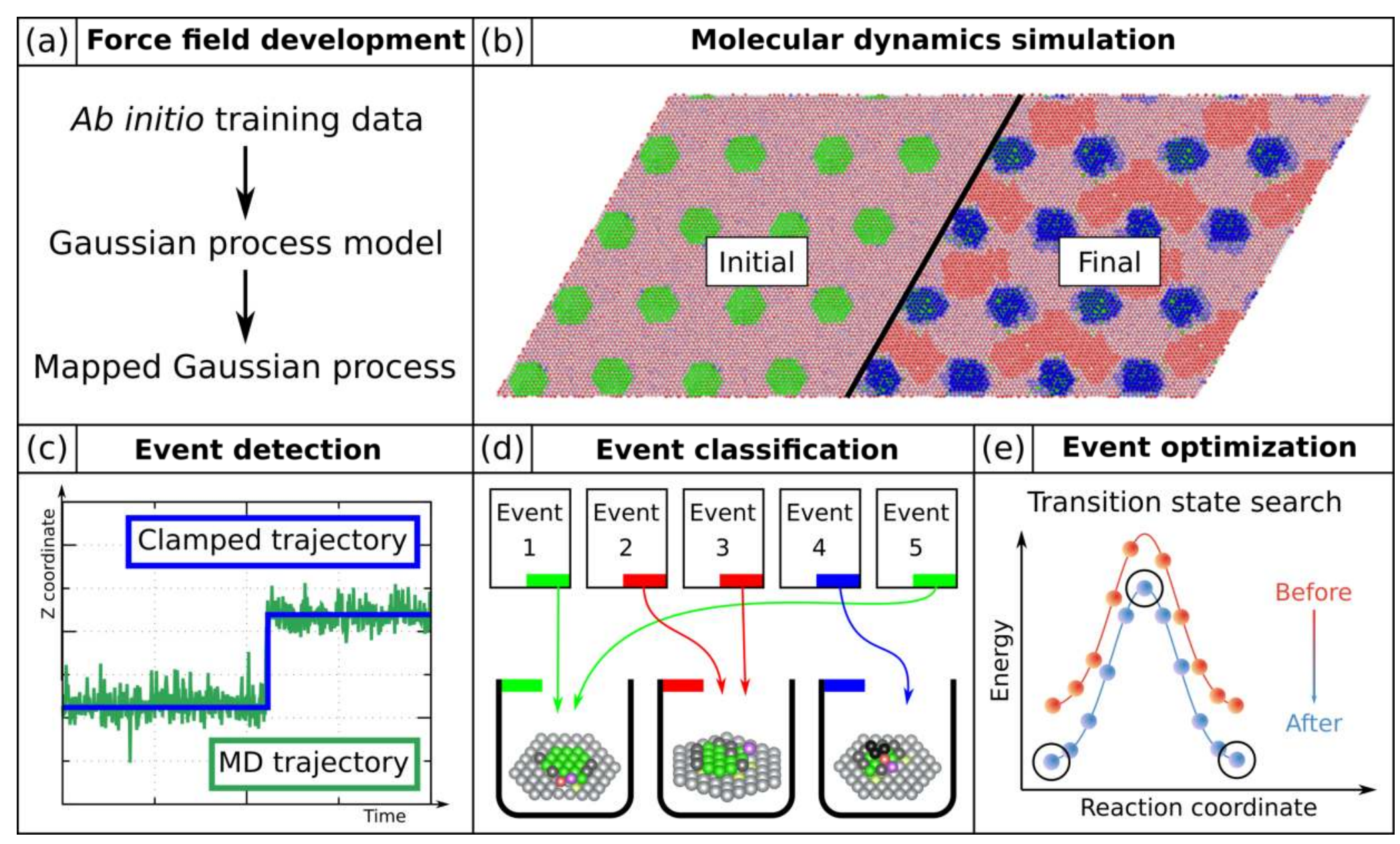




\section{$2 \mu \mathrm{s} @ 500$ K}

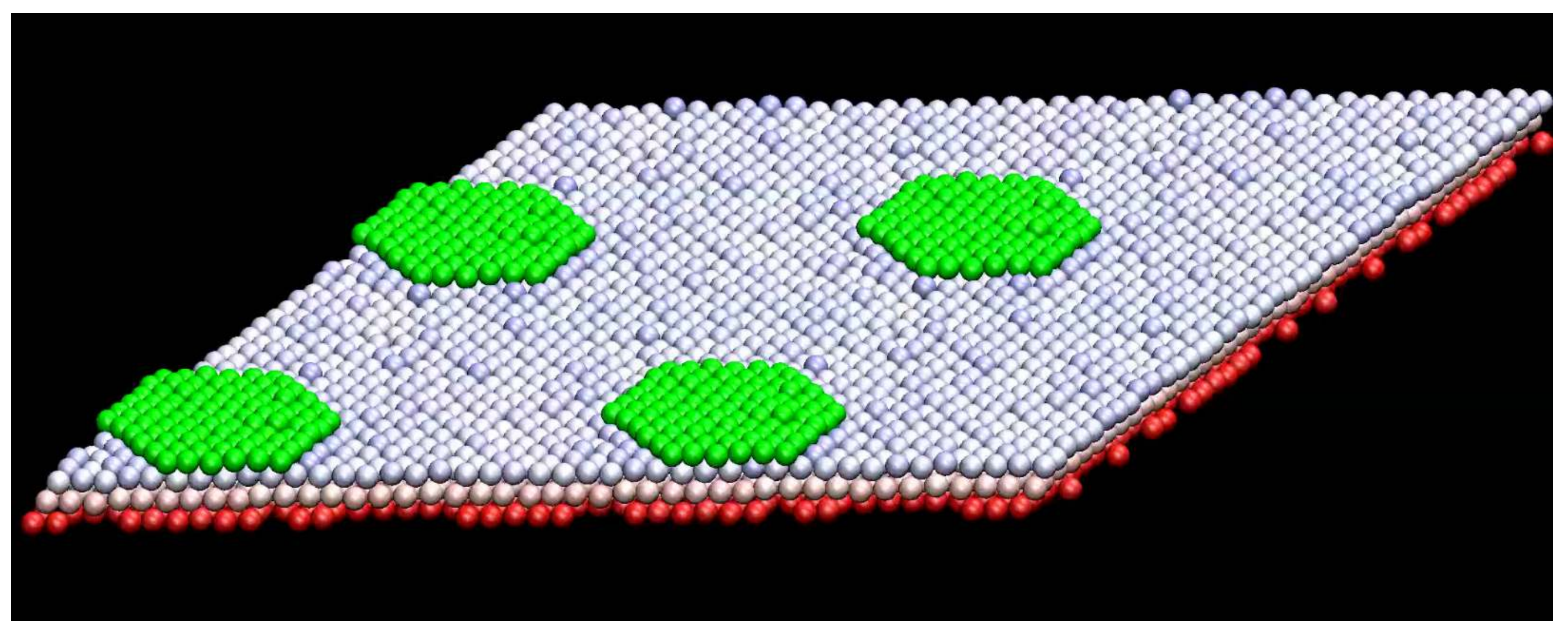

See video at: DOI: 10.6084/m9.figshare.11816832 (2020).

1) $\mathrm{Pd}-\mathrm{Ag}$ exchange around the edges

2) Formation of PdAg alloy layer @ Lv2

(4) $\begin{aligned} & \text { Materials } \\ & \text { Intelligence } \\ & \text { Research }\end{aligned}$

3) Ag encapsulation \& vacancy pit growth

파묨 HARVARD School of Engineering
and Applied Sciences 


\section{$0.1 \mu \mathrm{s} @ 900 \mathrm{~K}$}

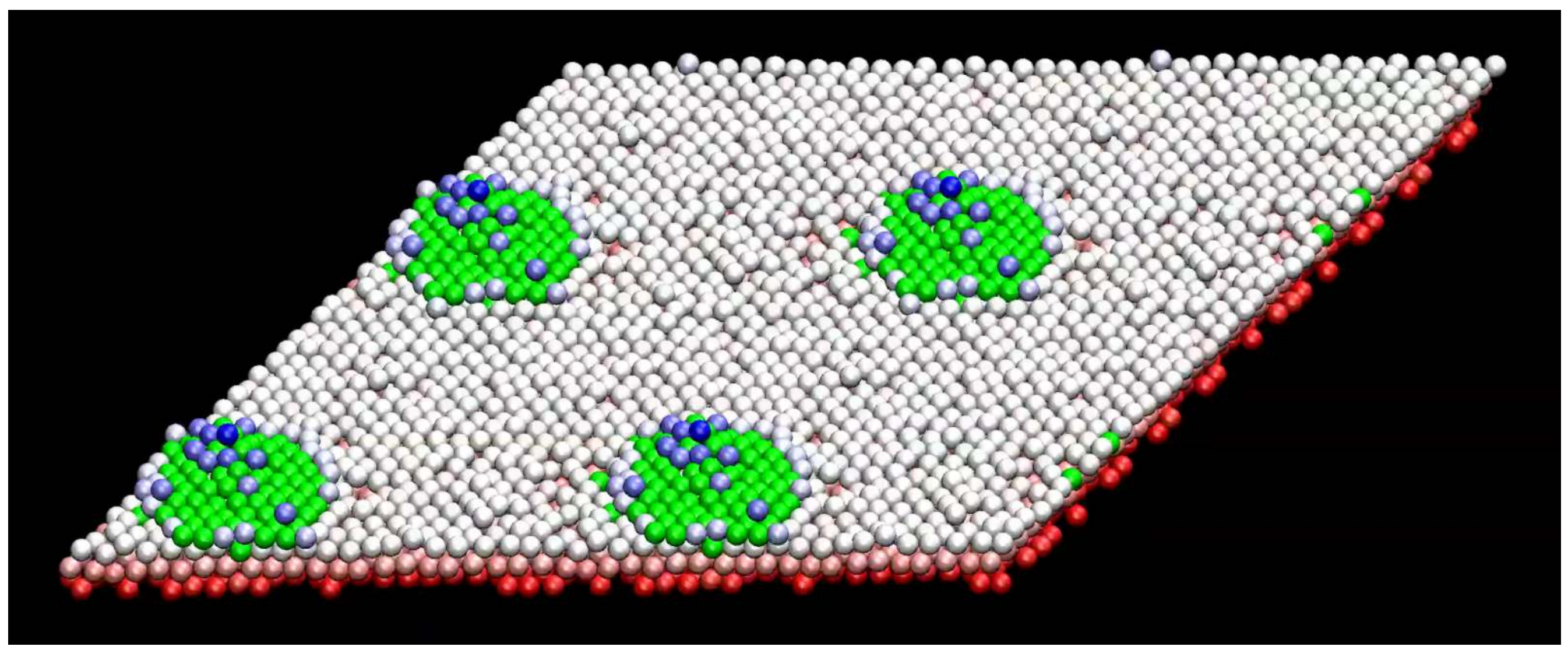

See video at: DOI: 10.6084/m9.figshare.11816847 (2020).

1) Ag encapsulation up to Lv3

2) Layer-by-layer dissolution \& vacancy pit decay 


\section{Restructuring events: $\mathrm{Pd} / \mathrm{Ag}$ heteroepitaxy}

IS

(a)

IS

(b)

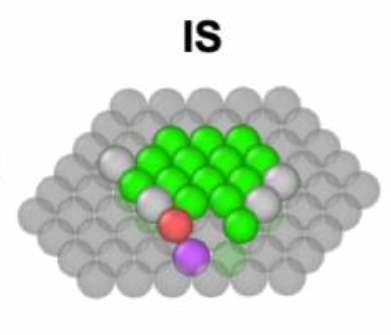

(c)

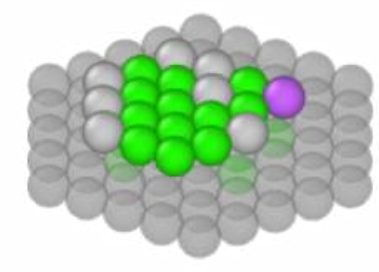

(d)

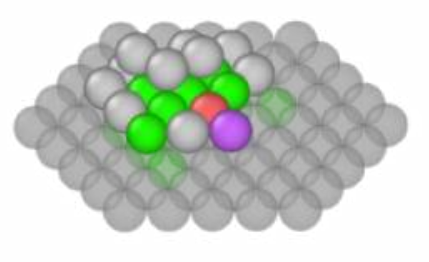

TS

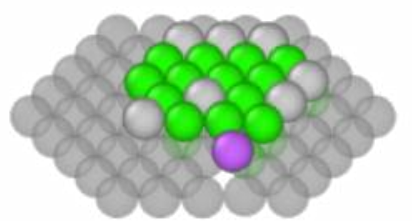

MS
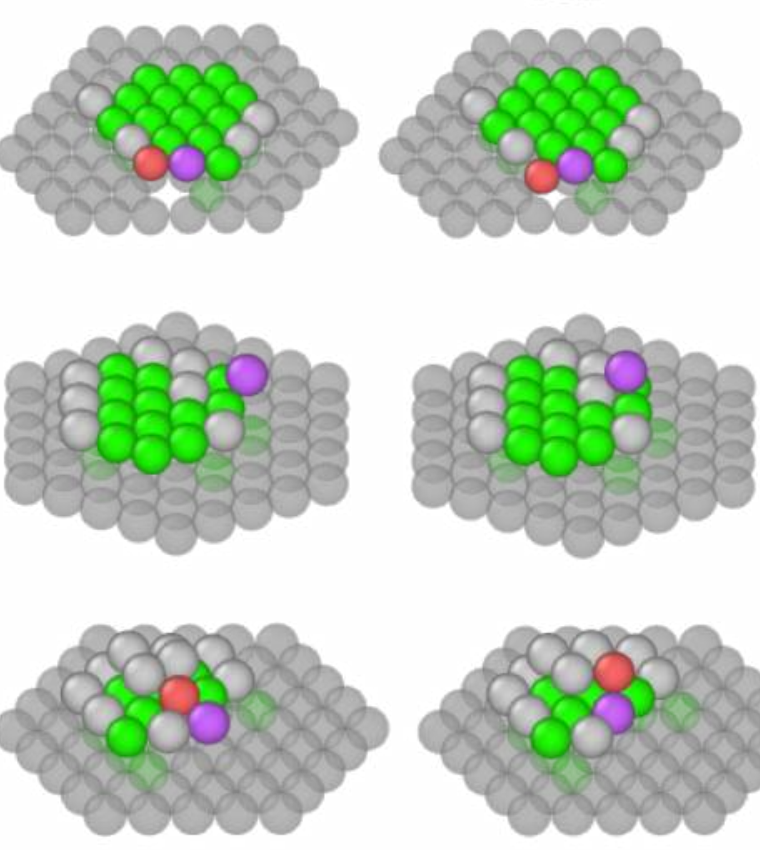

FS

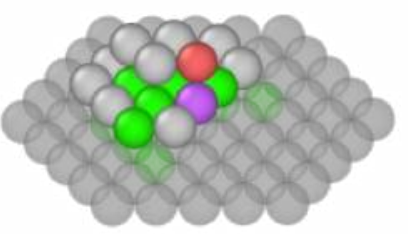

(a) Ag popout

(b) Pd-Ag direct exchange

(c) Ag hopping ascent

(d) Pd exchange ascent 


\section{Next investigations}

Deposition Annealing \& pretreatment

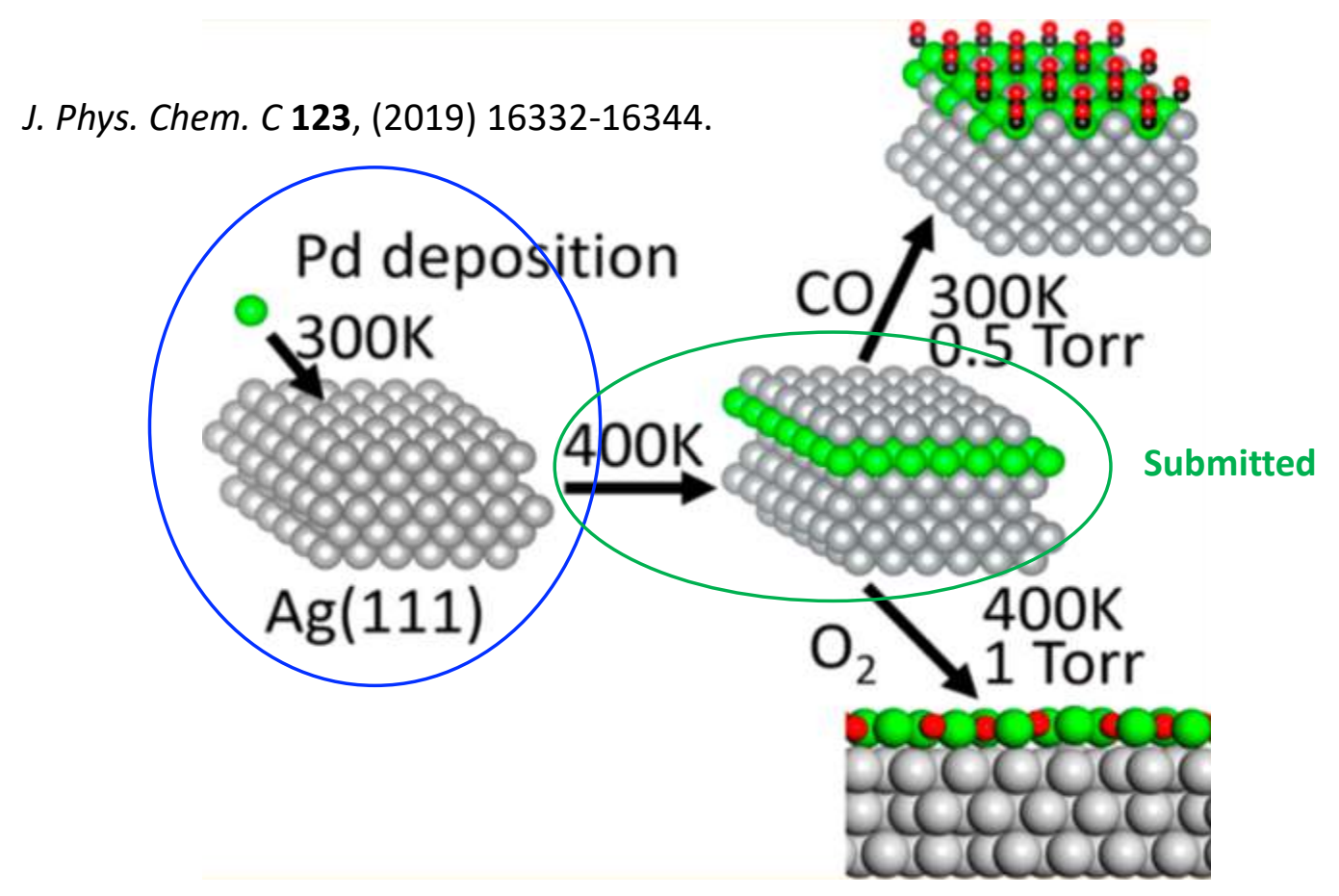

J. Phys. Chem. C 123, (2019) 8312-8323.
$\mathrm{H}_{2}$ activation \& spillover

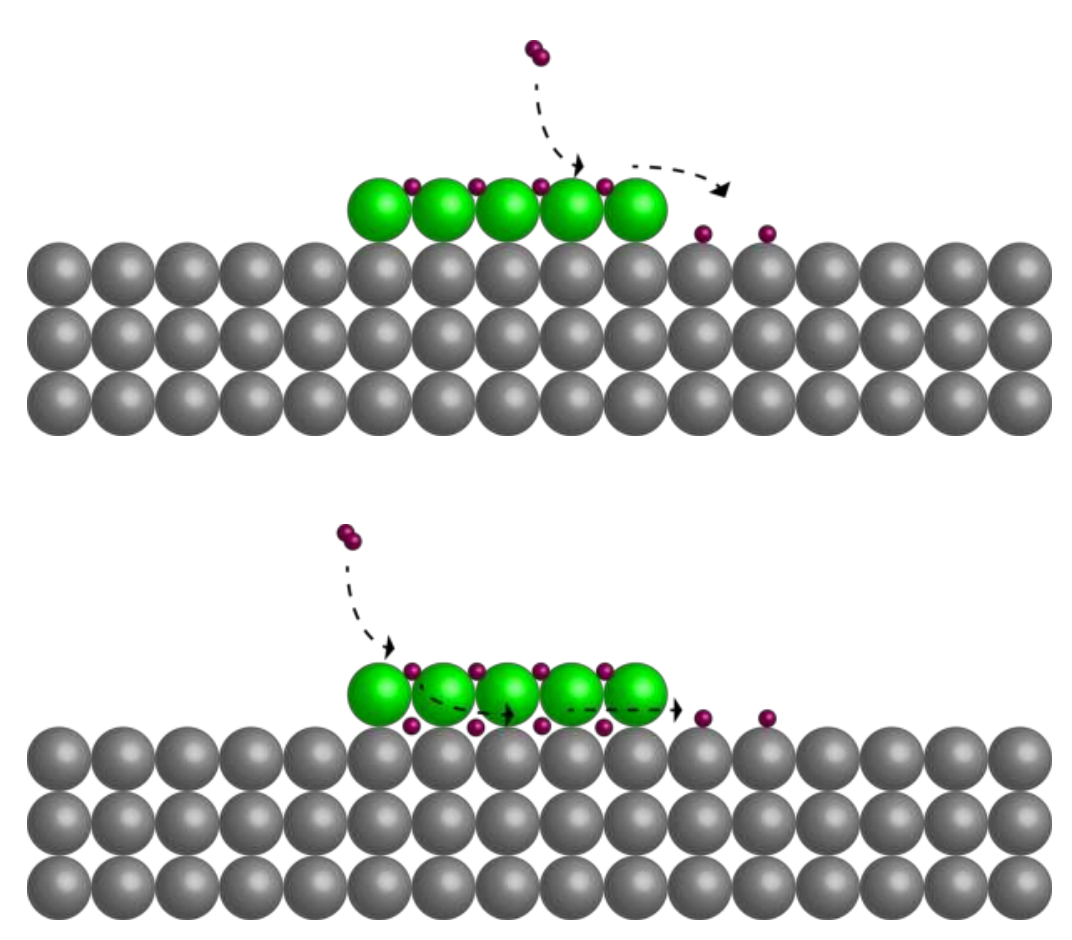

Undergoing 


\section{Acknowledgments}

- Integrated Mesoscale Architecture for Sustainable Catalysis (IMASC EFRC)

- Computational resources

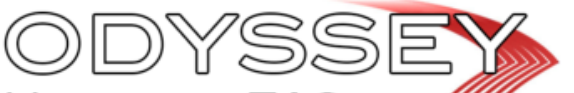
HARVARD FAS

RESEARCH COMPUTING

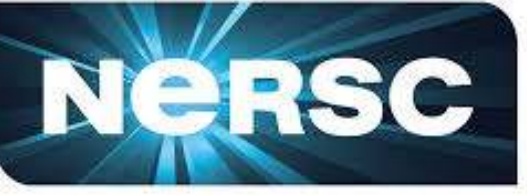

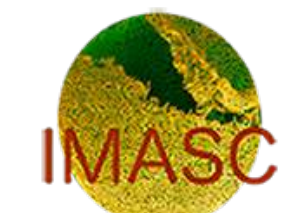
ar.

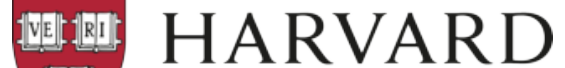
UNIVERSITY
- Advisors

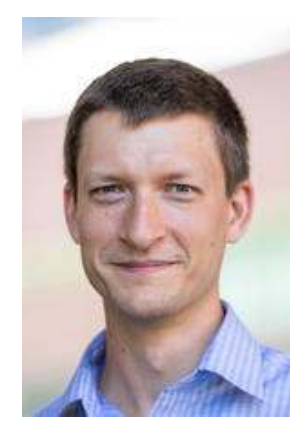

Prof. Boris Kozinsky Prof. Cynthia M. Friend

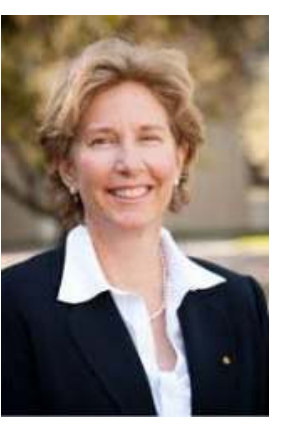

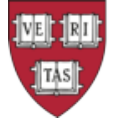

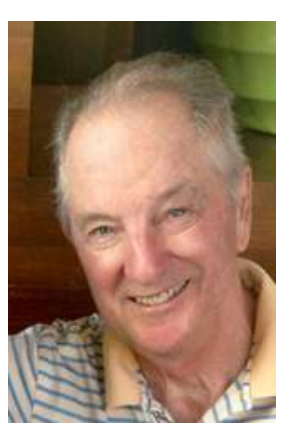

Prof. Robert J. Madix

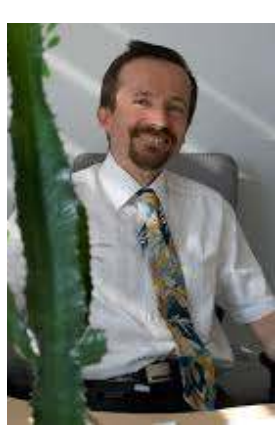

Prof. Philippe Sautet

UCLA

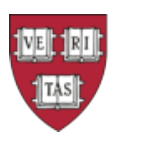

- Collaborators

Workflow \& simulation
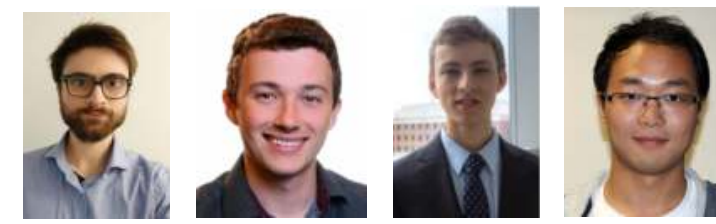

Dr. Nicola

Steven B.

Jacob Florian Duanmu

Method development
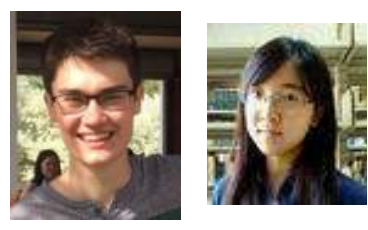

Jonathan Vandermause

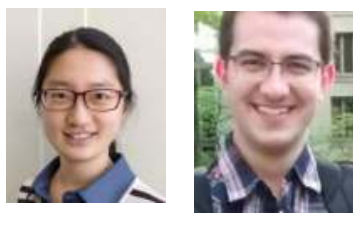

Yu Xie

Dr. Lixin

Albert Musaelian
XSEDE Extreme Science and Engineering
Discovery Environment

Surface science experiments

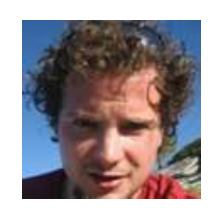

Dr. Matthijs A. van Spronsen
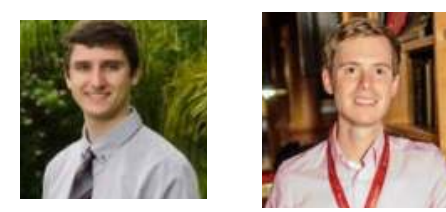

Christopher R O'Connor

Tobias Egle 


\section{Gaussian process: Forces}
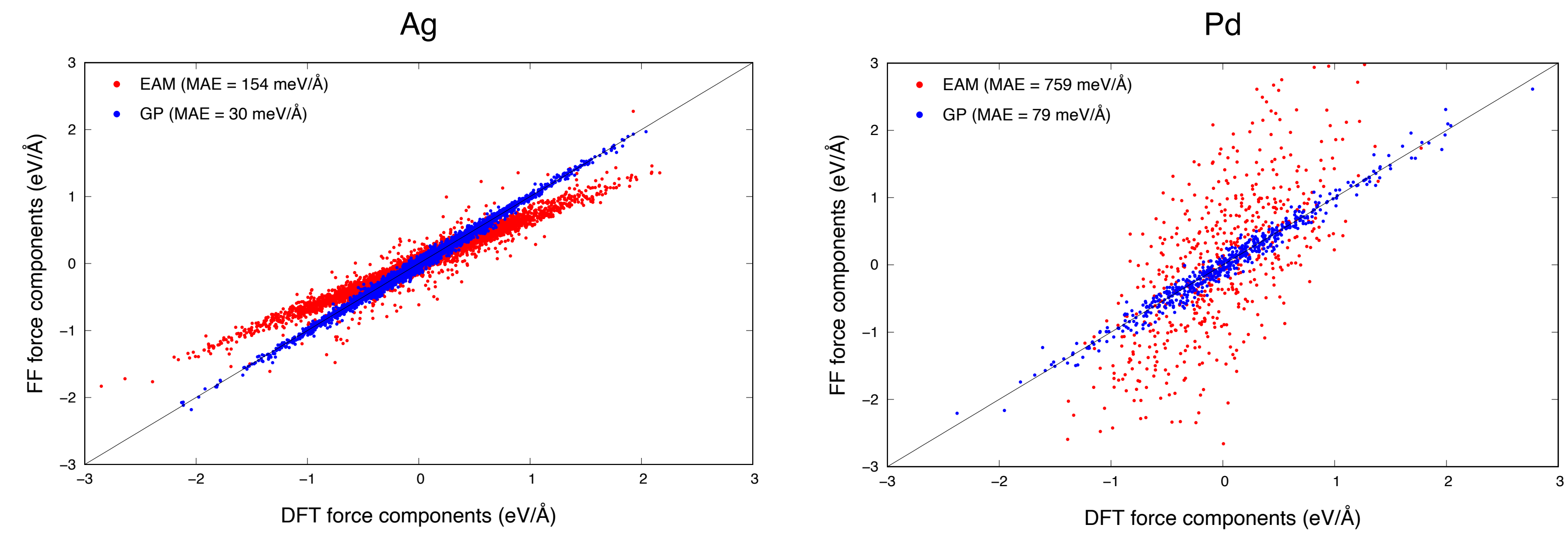

10 frames $\times 181$ atoms $\times 3$ components $=5430$ data points 


\section{Gaussian process: Energetics}

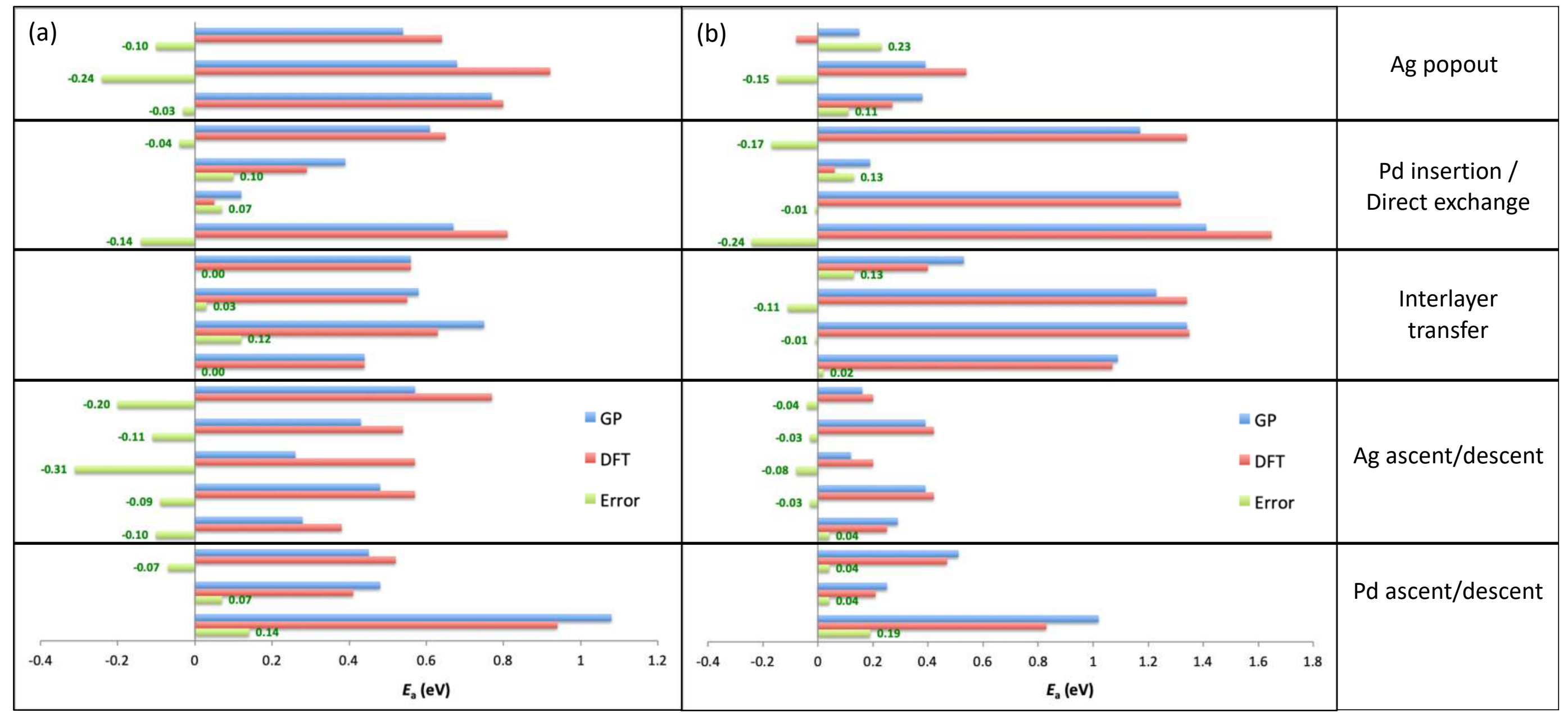

Materials

Intelligence

Research

\section{Activation energy}

$\mathrm{MAE}=0.10 \mathrm{eV}$ (0.4 meV/atom)
팔 HARVARD

School of Engineering and Applied Sciences 


\section{Event energetics}

IS

(a)

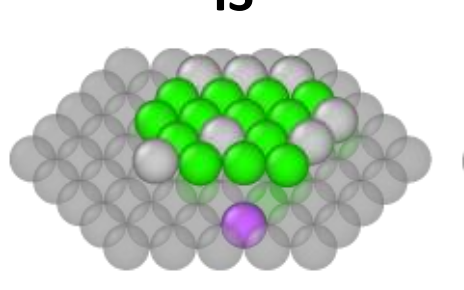

IS

(b)

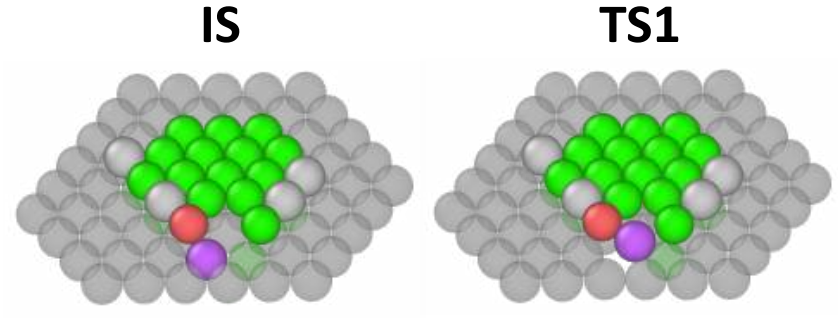

(c)

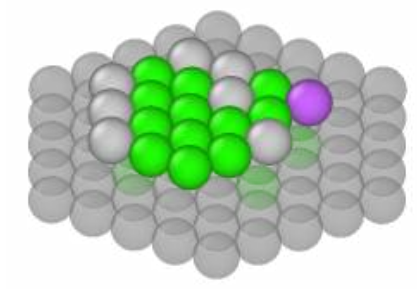

(d)

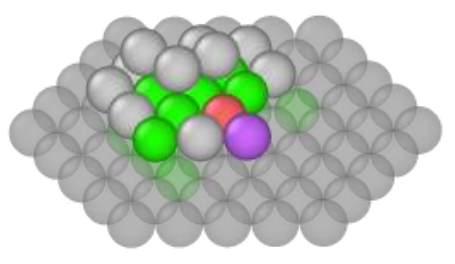

TS

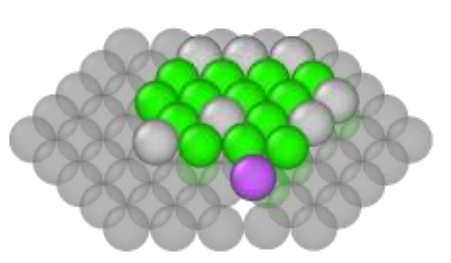

FS

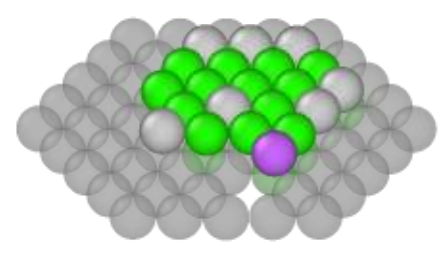

MS
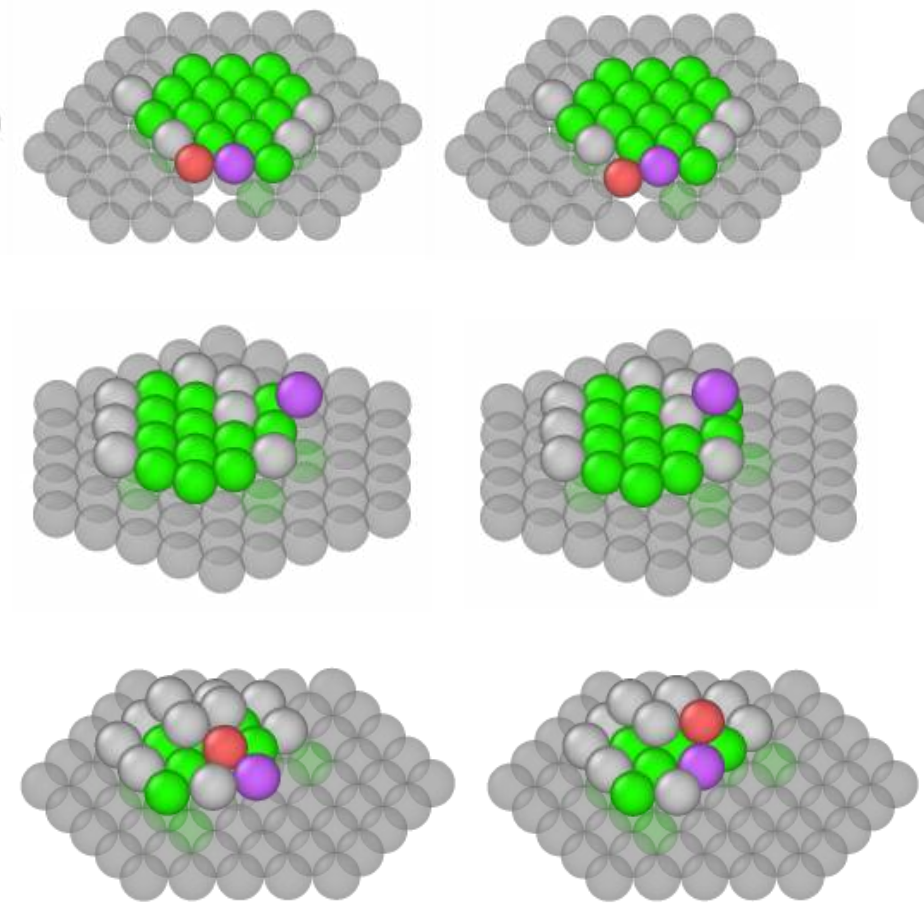

\section{Energy (eV)}

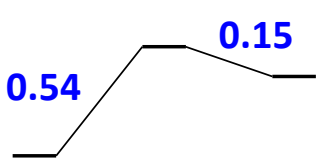

FS
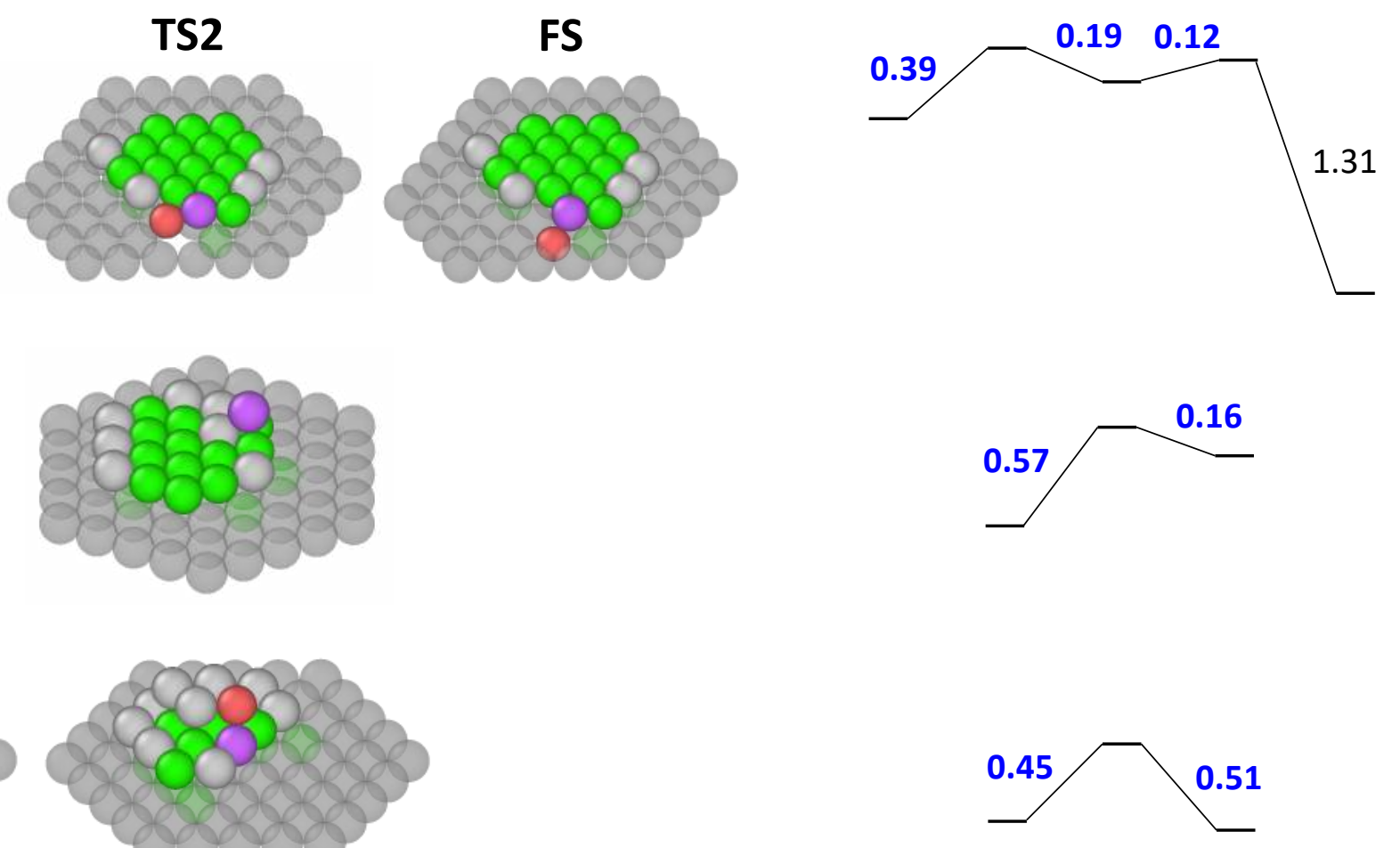

(4) $\begin{aligned} & \text { Materials } \\ & \text { Intelligence } \\ & \text { Research }\end{aligned}$

○ 9 Pd
Ag in motion $P d$ in motion
땀ㅁ HARVARD

School of Engineering
and Applied Sciences 


\section{Clamping vs. standard coordination number methods}

- Clamped: Hard cutoff of $3.0 \AA$ on the trajectory clamped to reference sites via "atomic democracy"

- Simple: Hard cutoff of $3.6 \AA \AA$ on the raw trajectory

\section{- Brunner (1977):}

1) Sort nearest neighbors within a large cutoff of $6.0 \AA$

2) They are at distances $d_{1}, d_{2}, \ldots$

3) Find maximal $d_{i+1} / d_{i}$

4) $d_{1}, \ldots, d_{i}$ are taken as the coordinating neighbors

\section{- Voronoi:}

1) Hard cutoff of $4.3 \AA$

2) Must share a Voronoi facet
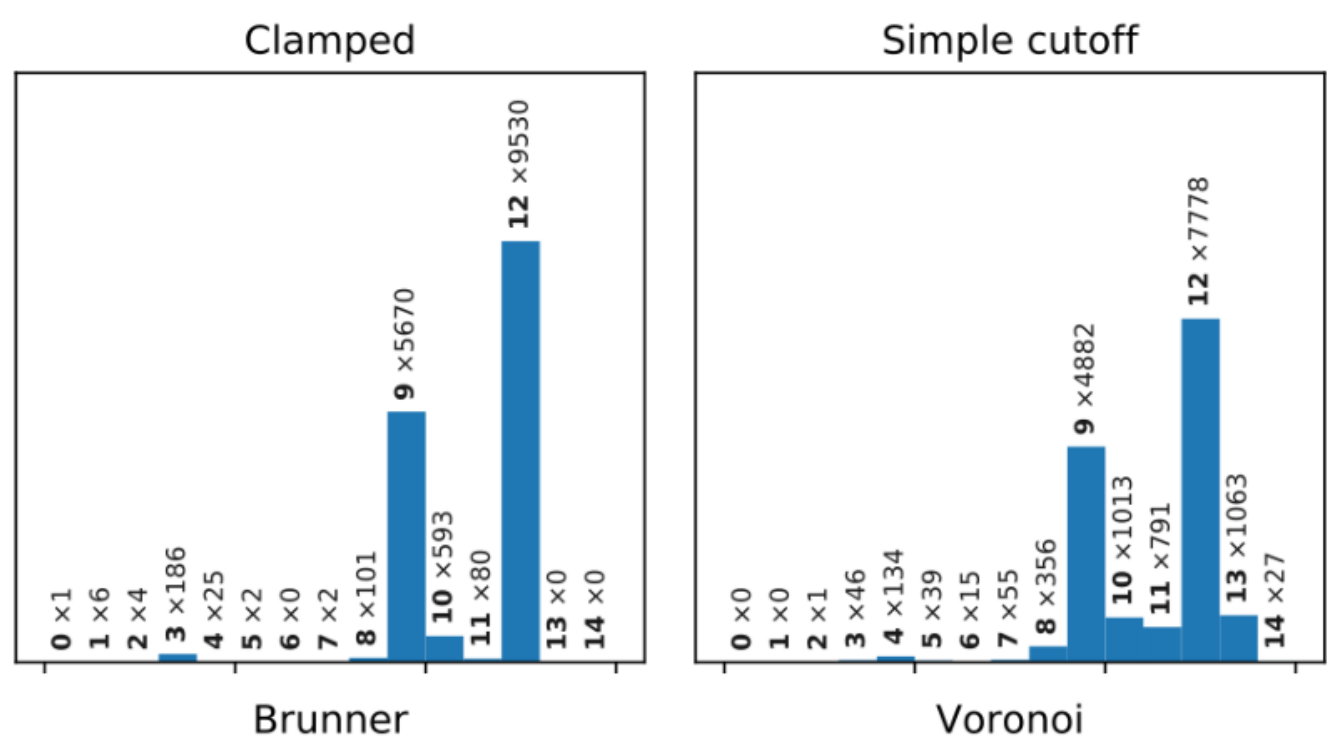

Brunner

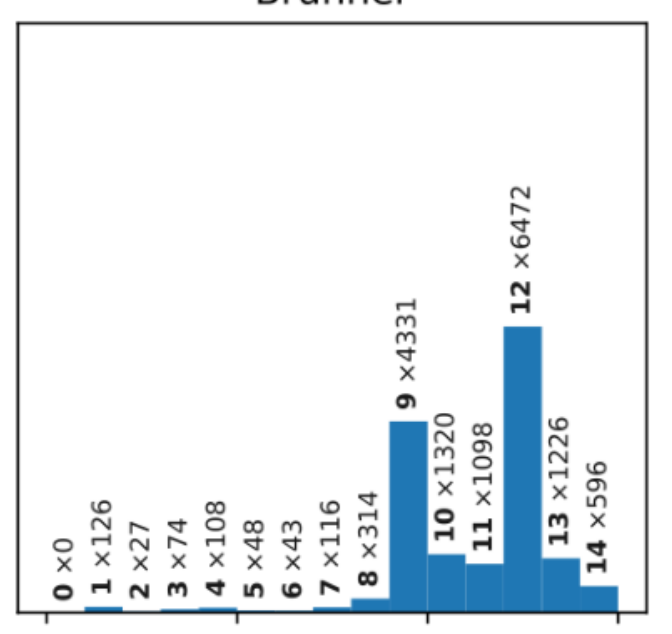

Voronoi

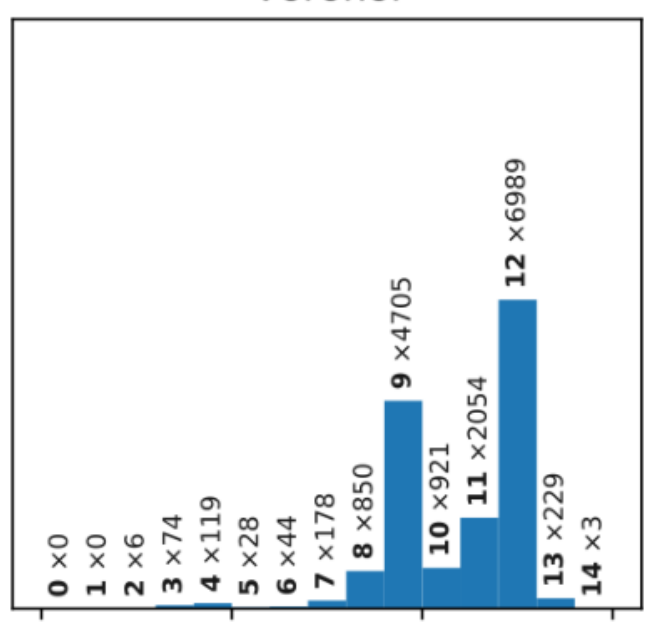




\section{Event classification}

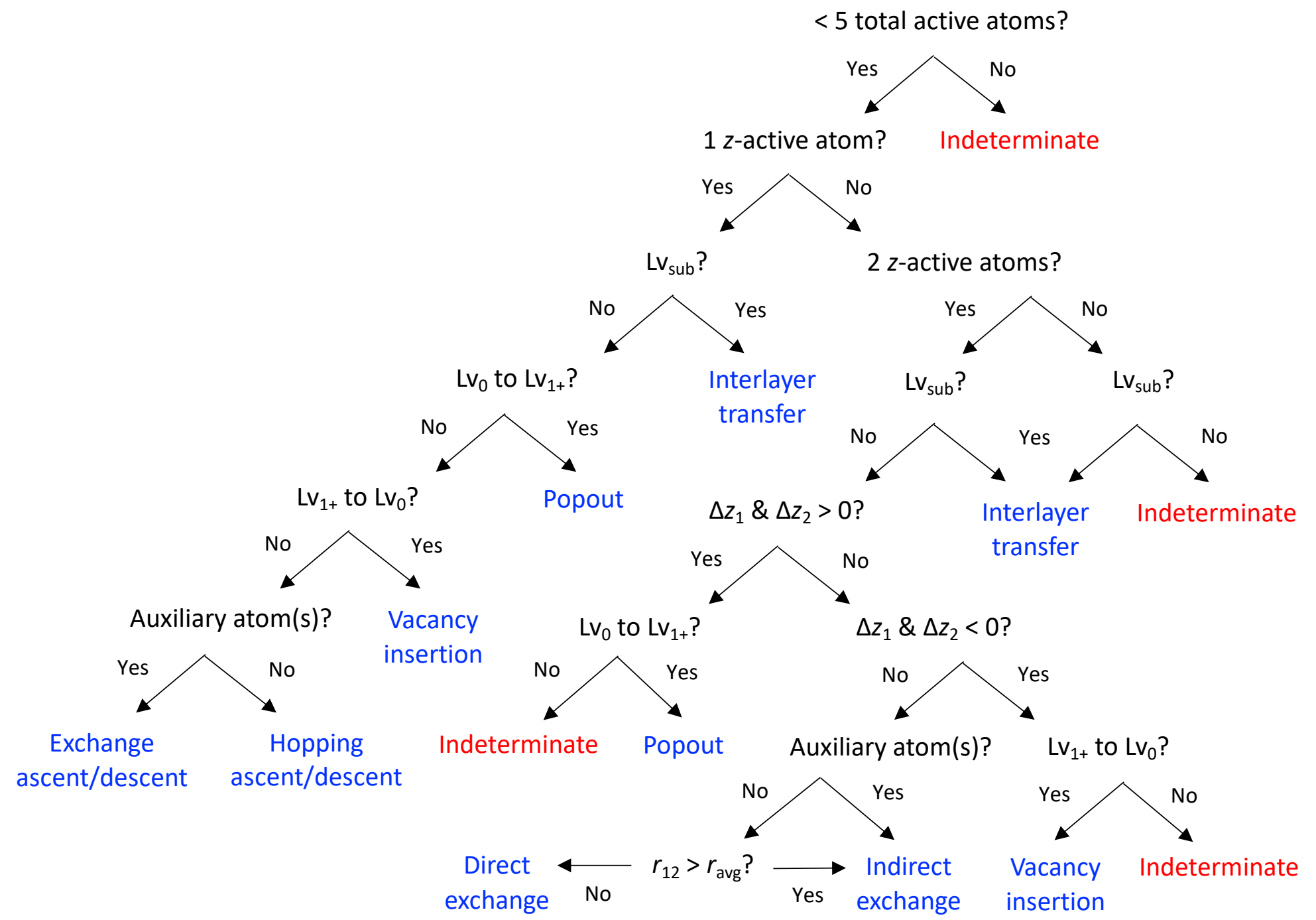




\section{Event statistics}
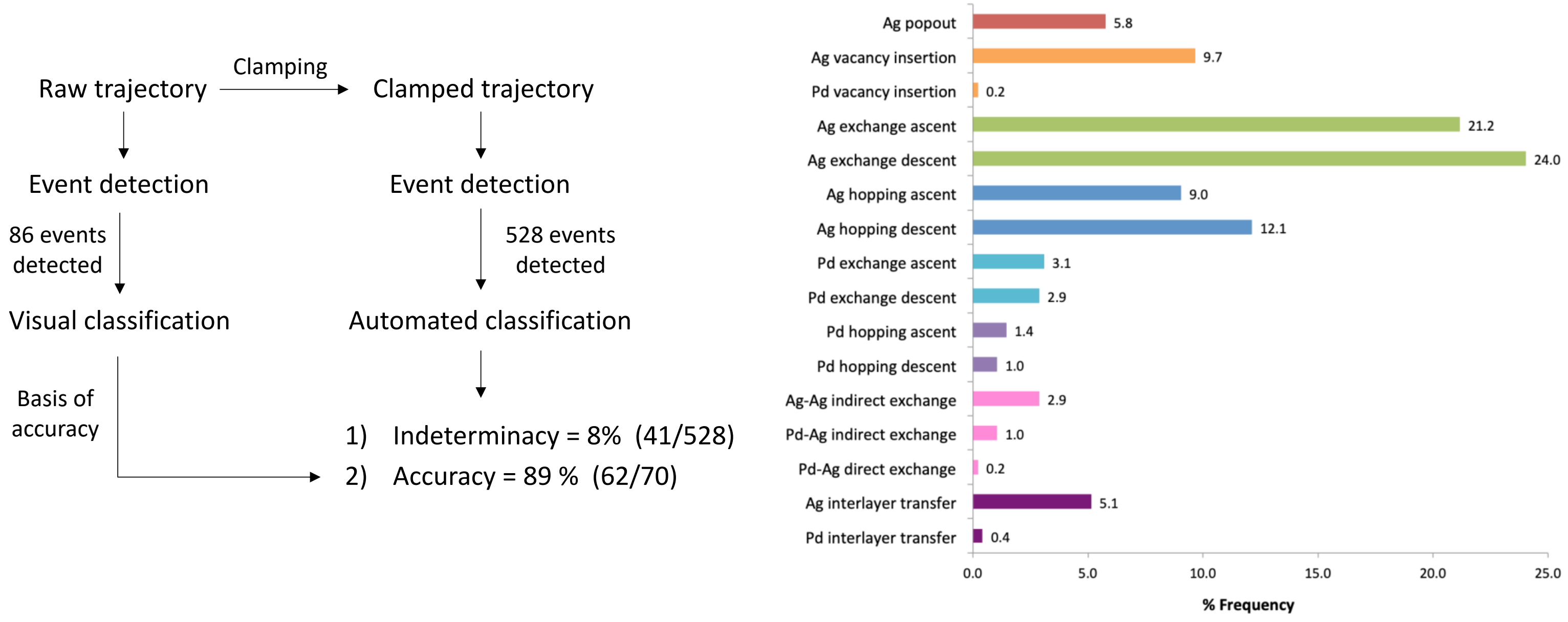


\section{CO probe experiments}

High resolution electron energy loss spectroscopy (HREELS)

A

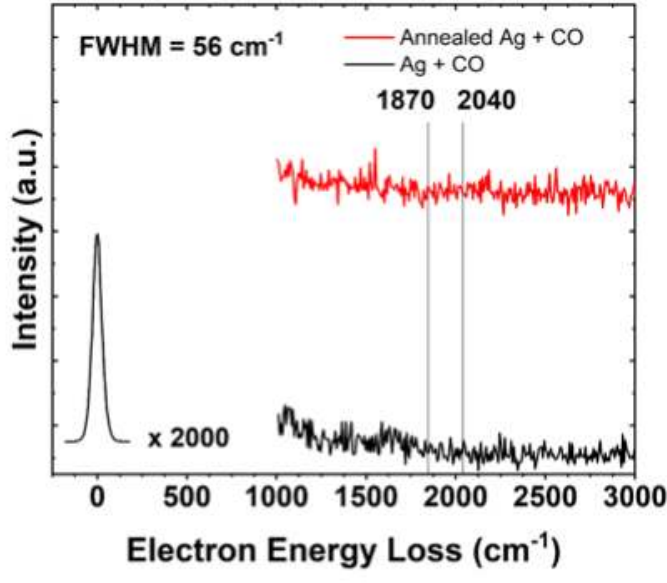

B

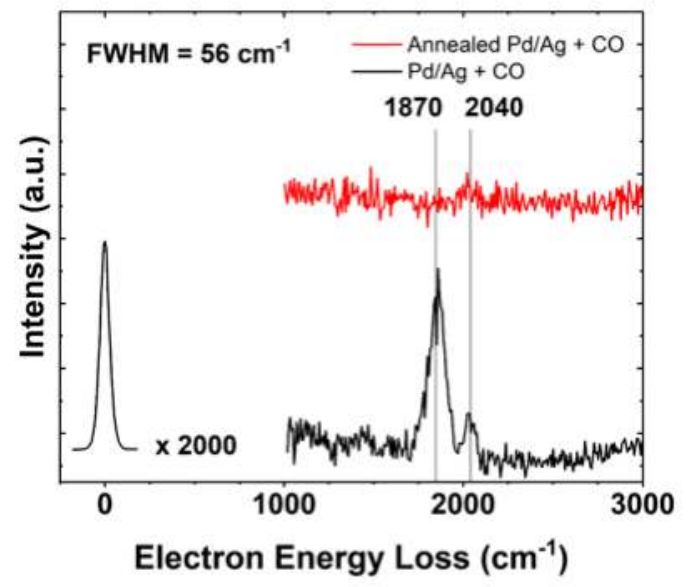

X-ray photoelectron spectroscopy (XPS)

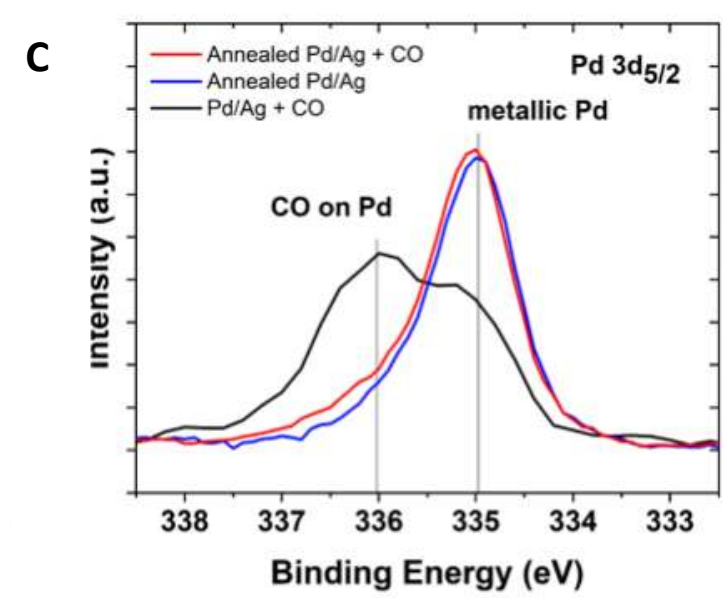

Low energy ion scattering spectroscopy (LEISS)

D

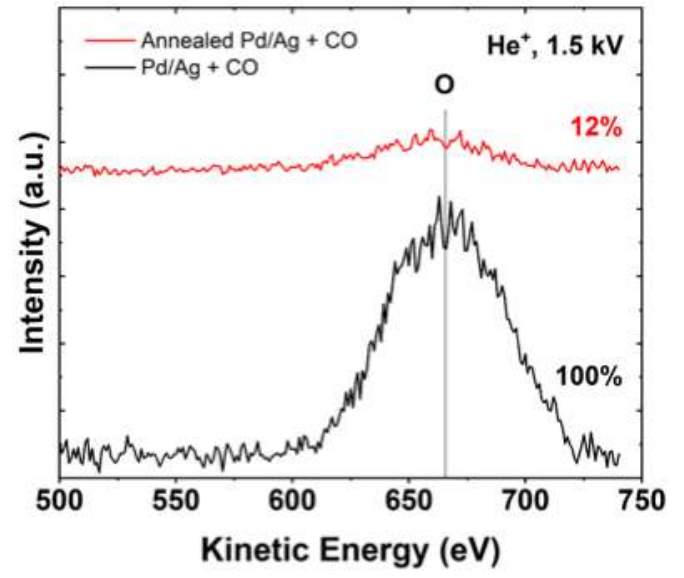

Temperature programmed desorption (TPD)

E

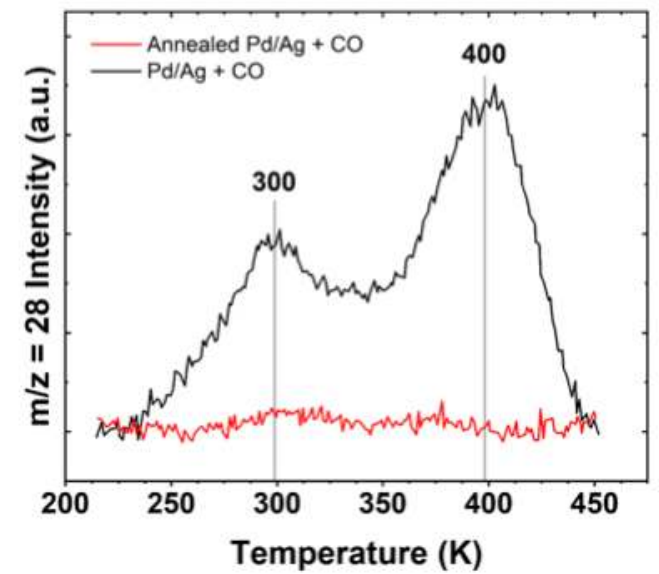

Materials

Intelligence

Research 


\section{Exchange-correlation functional benchmark}

(a)

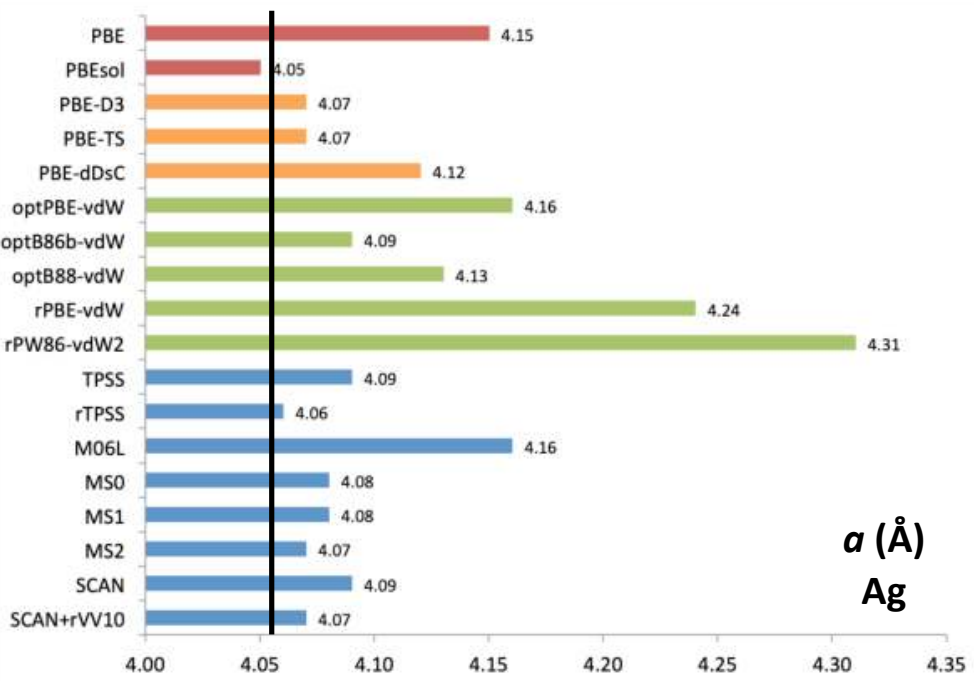

(b)

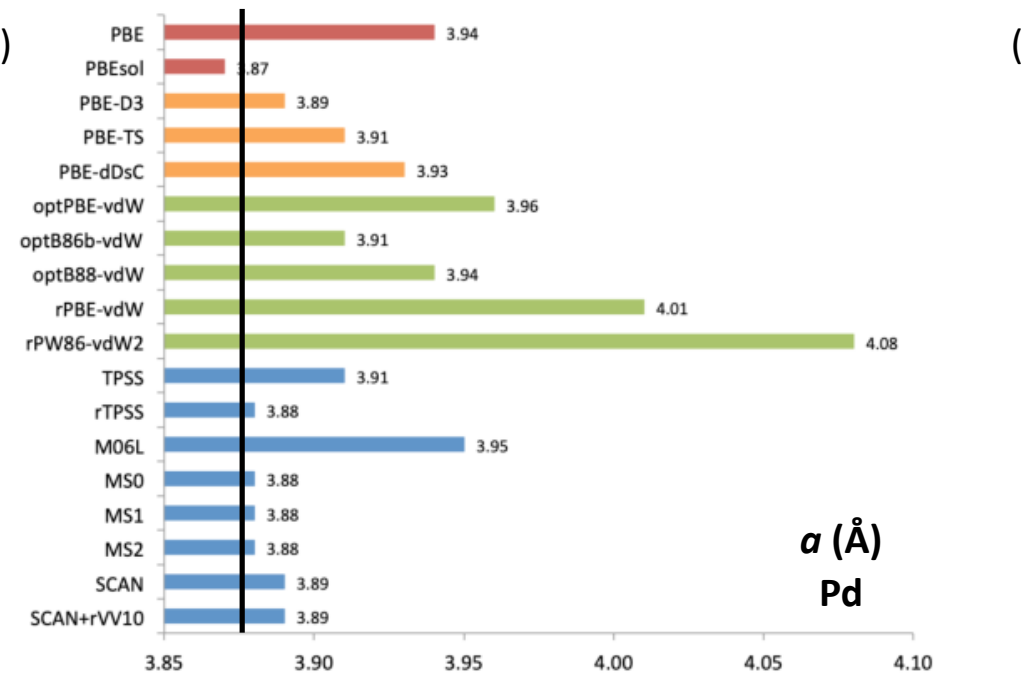

Materials

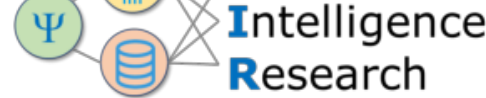

(4) Intelligenc (c)

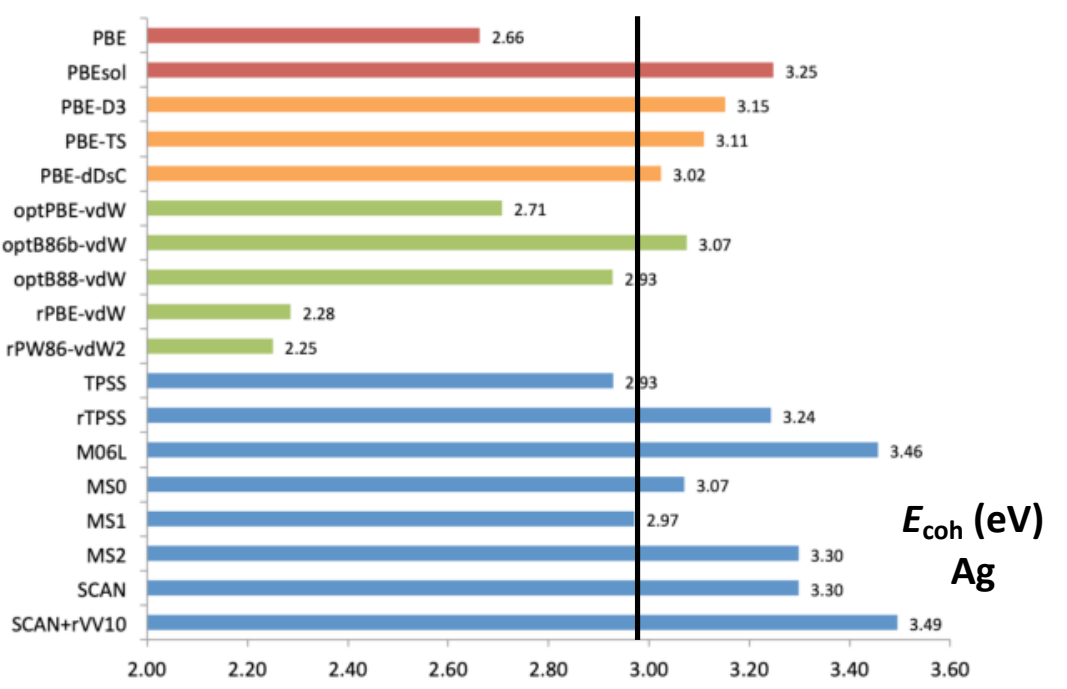

(d)

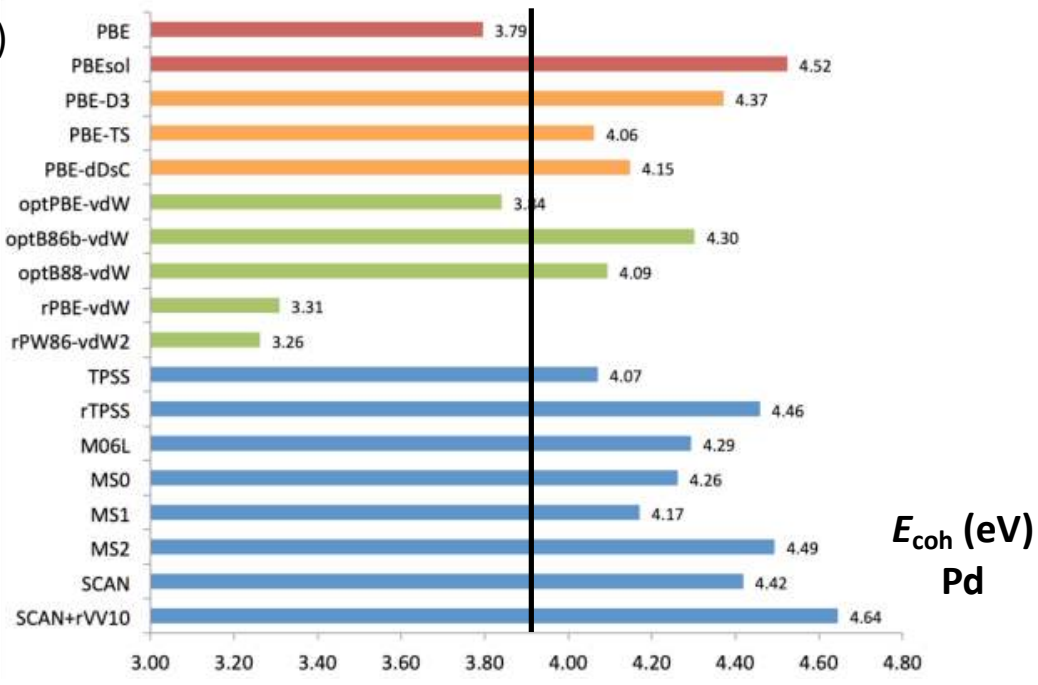

(e)

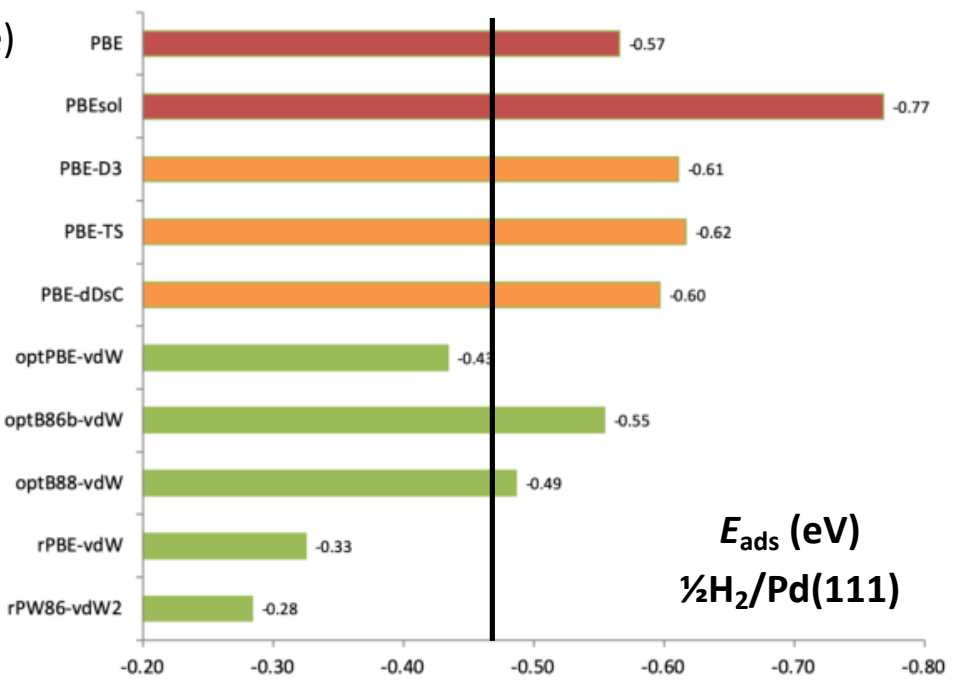

(f)

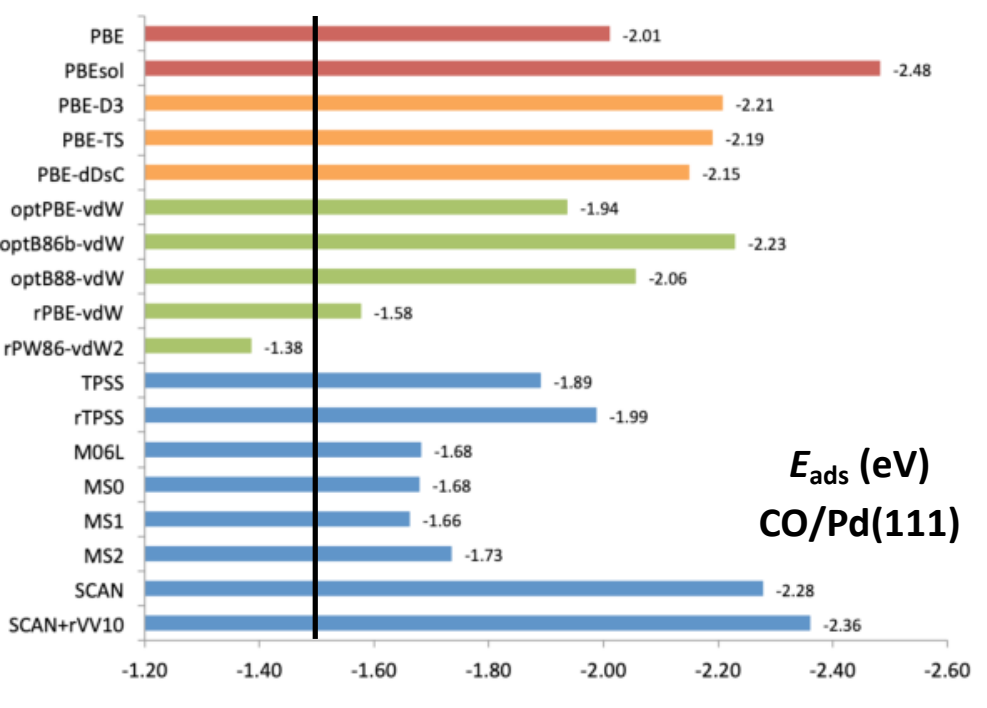




\section{Segregation energetics (M06L)}

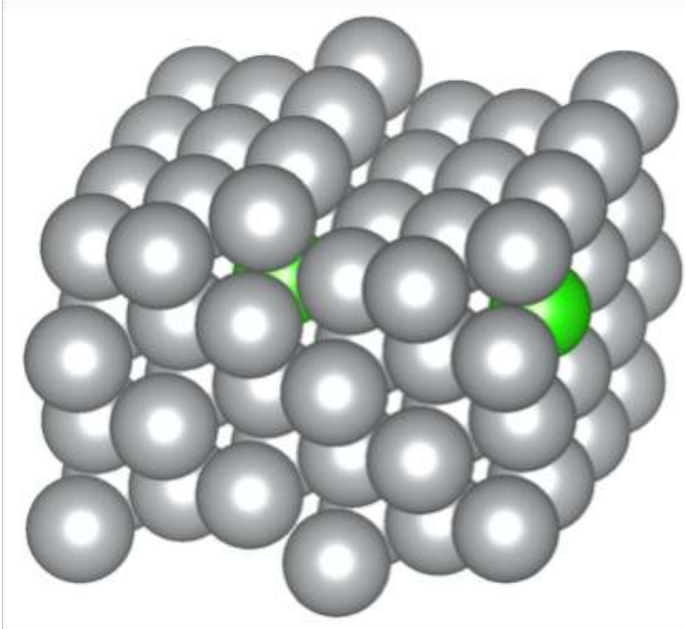

$E_{\text {seg }}=+0.42 \mathrm{eV}$

$E_{\mathrm{ads}}=-0.26 \mathrm{eV}$

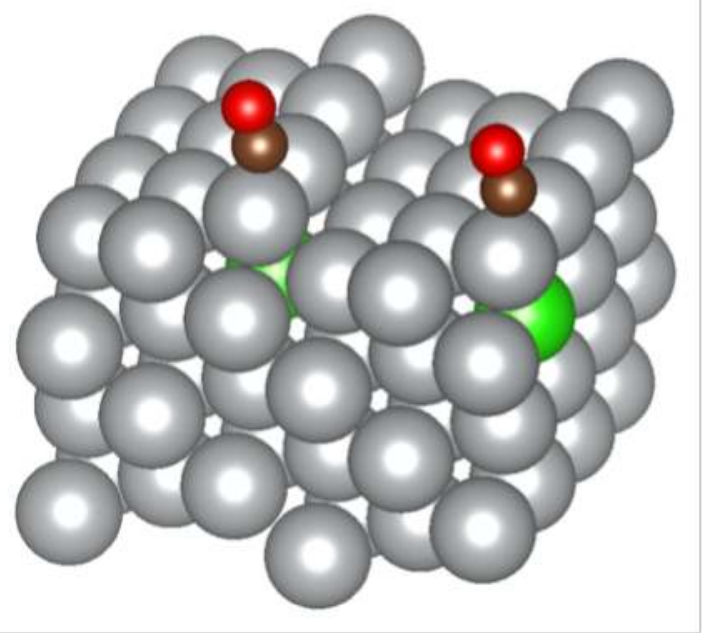

Ag

Pd

- C

- 0

$\stackrel{E_{\text {seg }}=-0.46 \mathrm{eV}}{\longrightarrow}$

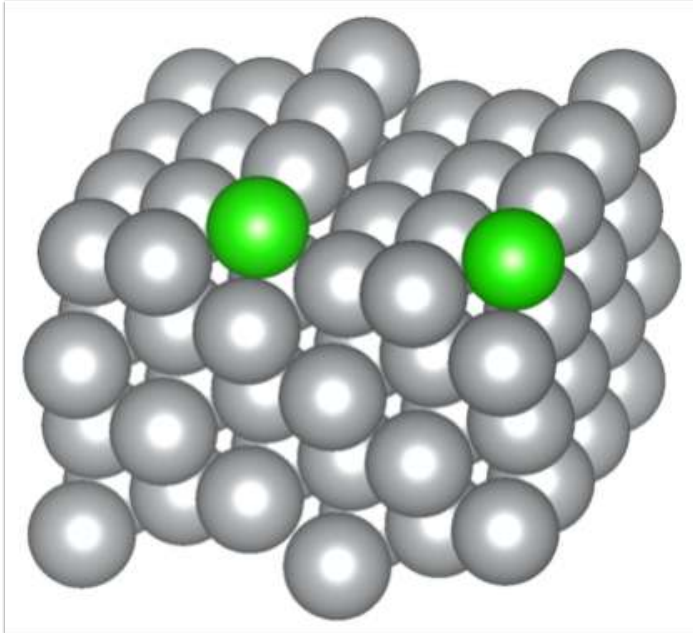

$\downarrow E_{\mathrm{ads}}=-1.14 \mathrm{eV}$

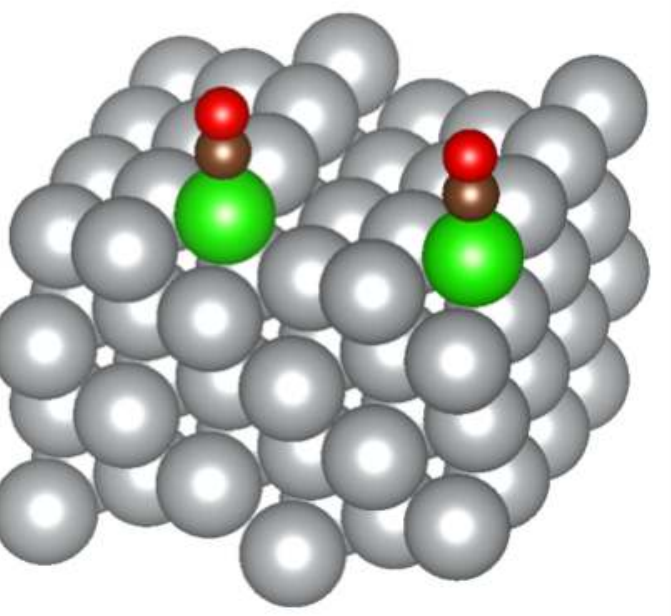

